\title{
Fast Min-hashing Indexing and Robust Spatio- temporal Matching for Detecting Video Copies
}

\author{
$\mathrm{CHIH}-\mathrm{YI} \mathrm{CHIU}$ \\ Institute of Information Science, Academia Sinica \\ HSIN-MIN WANG and CHU-SONG CHEN \\ Institute of Information Science, Academia Sinica \\ Research Center for Information Technology Innovation, Academia Sinica
}

\begin{abstract}
The increase in the number of video copies, both legal and illegal, has become a major problem in the multimedia and Internet era. In this paper, we propose a novel method for detecting various video copies in a video sequence. To achieve fast and robust detection, the method fully integrates several components, namely the min-hashing signature to compactly represent a video sequence, the spatio-temporal matching scheme to accurately evaluate the video similarity compiled from the spatial and temporal aspects, and some speed-up techniques to expedite both min-hashing indexing and spatio-temporal matching. The results of experiments demonstrate that, compared to several baseline methods with different feature descriptors and matching schemes, the proposed method that combines both global and local feature descriptors yields the best performance when encountering a variety of video transformation. The method is very fast, requiring approximately 0.06 seconds to search for copies of a thirty-second video clip in a six-hour video sequence.
\end{abstract}

Categories and Subject Descriptors: H.3.3 [Information Storage and Retrieval]: Information Search and Retrieval - Information filtering, Search process; I.2.10 [Artificial Intelligence]: Vision and Scene Understanding - Video analysis

General Terms: Algorithms, Design, Experimentation, Performance

Additional Key Words and Phrases: Content-based copy detection, near-duplicate, histogram pruning

\section{INTRODUCTION}

With the rapid development of multimedia technologies, digital videos are now ubiquitous on the Internet. According to a report by AccuStream iMedia Research (http://www.accustreamresearch.com), in 2006, the quantity of video streams increased $38.8 \%$ to 24.92 billion in media sites world-wide. One of the most popular video sharing sites, YouTube, hosted about 6.1 million videos, and 65,000 video clips were uploaded everyday. The enormous growth in the amount of video data has led to the requirement for efficient and effective techniques of video indexing and retrieval. In particular, since digital videos can be easily duplicated, edited, and disseminated, video copying has become an increasingly serious problem. A video copy detection technique would thus be helpful for protecting and managing video content. For instance, with such a technique,

Some parts of this work were published in Chiu et al. [2007].

This work was supported in part by the Taiwan e-Learning and Digital Archives Program (TELDAP), sponsored by the National Science Council of Taiwan under Grants: NSC 96-3113-H-001-011 and NSC 96-3113-H001-012.

Authors' addresses: C. Y. Chiu, H. M. Wang, and C. S. Chen: Institute of Information Science, Academia Sinica, 128 Academia Road, Section 2, Nankang, Taipei 115, Taiwan.

(C) 2008 ACM 1073-0516/01/0300-0034 \$5.00 
content providers could track particular videos with respect to royalty payments and possible copyright infringements. Platform providers could remove identical copies uploaded by users or aggregate near-duplicate videos into groups to facilitate browsing. Fig. 1 shows the result of inputting the phrase "UFO Apollo 11" to YouTube. Several sets of identical or near-duplicate video clips are displayed on the search result page. If the nearduplicates of the same set are compacted into a single item, the search result page can display more diverse video clips for users.

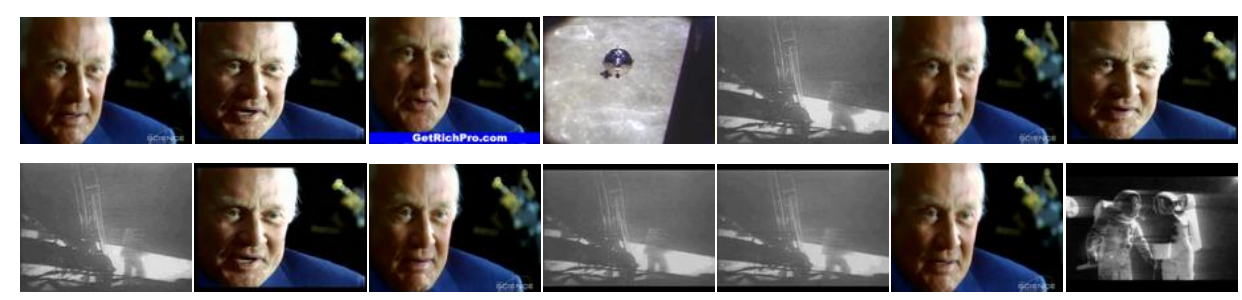

Fig. 1. The first fourteen search results retrieved by inputting the phrase "UFO Apollo 11" to YouTube. Most of the video clips are identical or near-duplicates, which can be removed or aggregated to facilitate user browsing.

There are two general techniques for detecting video copies: digital watermarking and content-based copy detection. Digital watermarking embeds hidden information in video, while the content-based technique employs perceptual features of the video content as a unique signature to distinguish one video from another. Because the latter does not destroy or damage video content, it has generated a great deal of research interest recently. Most existing content-based methods treat a video as a set of individual image frames and focus on the investigation of compact image features or efficient index structures. However, this confines their use to particular applications. For example, some methods are limited to dealing with certain types of video transformation, while others are limited to other types depending on the properties of the features they use. Besides, since these methods seldom consider time-related features, they have difficulty handling video transformation types that modify the temporal structure of video, such as slow motion and frame insertion/deletion. The pre-built index structures also cause an inherent search limitation, as some methods need to partition a video sequence into basic index units (e.g., shots) in advance. These methods might be inappropriate for monitoring broadcast streams.

In this paper, we address the limitations posed by existing methods. We classify various video copies into four categories with respect to the spatial and temporal transformation. While current methods are limited to handling one or two categories, we propose a 
novel video content analysis scheme, called spatio-temporal matching, to tackle all the categories in a unified manner. The proposed matching scheme, which analyzes the content similarity of two videos compiled from the spatial and temporal aspects, can effectively handle a variety of video transformation.

To avoid the limitation caused by the pre-built index structures, we employ a sliding window approach that would be more appropriate for monitoring broadcast streams. Without the index structures, however, the search time spent on a large-scale video sequence would be considerable. To make the search more efficient, the proposed method adopts a coarse-to-fine matching strategy. In the coarse stage, the sliding window scans the entire sequence to find potential candidate clips quickly. Each windowed sequence, as well as the given query clip, is represented by a novel feature called the min-hashing signature that is compact and robust. For example, in this study, a 30-dimensional signature is sufficient to represent a sixty-frame sequence. In the fine stage, the above-mentioned spatio-temporal matching is used to filter out false positives from the candidate set. In addition, some speed-up techniques are applied in both stages with great effect; hence, overall, the proposed method is very fast.

To evaluate the proposed method, we implement several baseline methods that use different feature descriptors (e.g., the ordinal measure and the SIFT descriptor), as well as different matching schemes. The results of extensive experiments show that, with the composed feature descriptor, the proposed method achieves excellent results, which demonstrate the widest coverage for various transformation types among all compared methods. The proposed method is also very efficient, as it only needs about 0.06 seconds to search for copies of a thirty-second query clip in a six-hour video sequence.

The remainder of this paper is organized as follows. In Section 2, we formulate the video copy detection problem. Section 3 reviews recent work related to video copy detection. In Section 4, we detail the proposed method, including min-hashing indexing and spatio-temporal matching. Section 5 describes the extensive evaluation experiments. Then, in Section 6, we summarize our conclusions.

\section{PROBLEM FORMULATION}

Let $Q$ be a query clip with $n$ frames, and $T$ be a target sequence with $m$ frames, where $n$ $<<m$. Here we assume that $Q$ is an original video source and $T$ is a suspect video stream collected from a Web or TV broadcast. Suppose that in $T$, there exists a subsequence $C$ that is a copy derived by applying some types of video transformation (e.g., noise addi- 
tion and compression) to $Q$. Our goal is to locate $C$ in $T$ for the given $Q$ quickly and accurately.

If $C$ is a copy of $Q$, a certain portion of their contents will be perceptually similar, where the "portion" is a subjective factor that varies case by case. Although we can evaluate the content similarity of $C$ and $Q$ directly, video copy detection is still challenging in terms of effectiveness and efficiency.

Let us consider the effectiveness issue. Since several types of video transformation can be applied to $Q$, the design of the similarity measure becomes complicated when it has to handle various video copies. The video transformation types can be classified into four basic categories according to their spatial and temporal characteristics:

(1) Whole region-preserved spatial transformation. This category includes brightness enhancement, histogram equalization, noise addition, compression, and frame resolution change. These transformation types modify the frame content while preserving the whole frame region, as shown in Fig. 2(b).

(2) Partial region-discarded spatial transformation. This category includes cropping and zooming in, which discard partial regions of a frame and modify the remaining content, as shown in Fig. 2(c).

(3) Frame number-changed temporal transformation. This category includes frame rate change and video speed change (fast forward and slow motion), which increase or decrease the number of frames without changing their order, as shown in Fig. 2(d).

(4) Frame order-changed temporal transformation. This category includes frame insertion/deletion/swap, which inserts, deletes, or swaps frames at any time point; thus, it changes the order of the source content, as shown in Fig. 2(e).

(b)

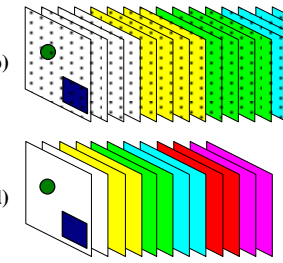

(a)

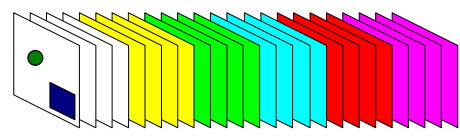

(c)

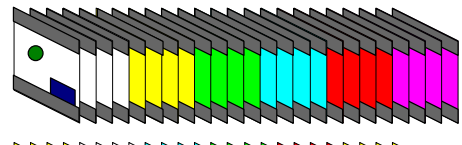

(e)

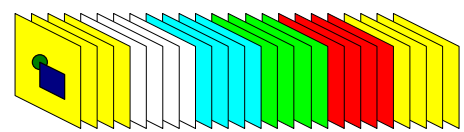

Fig. 2. A variety of video transformation types. Different colors are used to distinguish video subsequences: (a) the source video; (b) the transformed video derived by whole region-preserved spatial transformation (noise addition); (c) the transformed video derived by partial region-discarded spatial transformation (cropping); (d) the transformed video derived by frame number-changed temporal transformation (double-speed); and (e) the transformed video derived by frame order-changed temporal transformation (frame insertion/deletion/swap). 
Since we do not know the types of video transformation that have been applied to the source video in advance, a robust matching scheme is required to handle diverse video copies.

With regard to the issue of efficiency, if $T$ is a large-scale video sequence, (say, several hours or hundreds of hours), searching in $T$ will be very time-consuming. Generally, two search approaches can be used to address the efficiency issue. One is the partitioning and indexing approach, where $T$ is partitioned into basic index units, and then indexed into a tree or hash structure, as shown in Fig. 3(a). The index structure allows this approach to omit many unnecessary comparisons, thereby reducing the computation cost. However, its partitioning process not only requires extra computation, but also makes it difficult to monitor broadcast streams. Besides, this approach might lack the ability to locate the exact time position of the copied segment in $T$, if we do not maintain every frame's time code in an index unit.

The second approach uses a sliding window to scan the video sequence $T$ and computes the similarity between the given query clip and the windowed sequence. The window moves forward along $T$ for each similarity computation, as shown in Fig. 3(b). Compared with the partitioning and indexing approach, the merits of the window sliding approach include (1) its simple computation technique; (2) its ability to indicate the definite time position of a detected copy; and (3) its applicability to searching either a partitioned video dataset or an un-partitioned video sequence. In addition, there is a speed-up algorithm, which accelerates the window scanning procedure without sacrificing the search accuracy, making this approach fast enough in a large-scale video sequence.

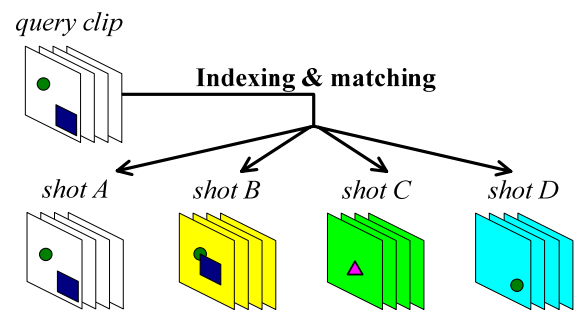

(a)

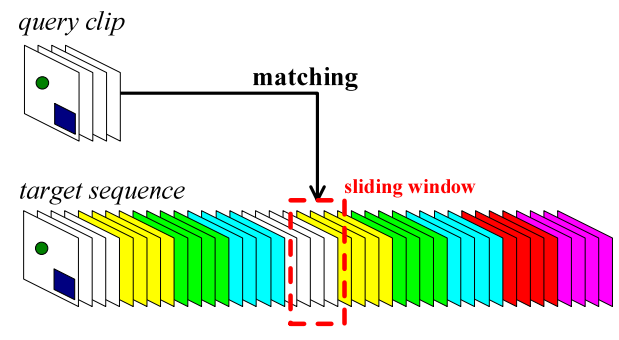

(b)

Fig. 3. Examples of the search approaches: (a) partitioning and indexing; and (b) window sliding.

\section{RELATED WORK}


Our study of the video copy detection problem begins with Content-Based Video Retrieval (CBVR). The general paradigm of the CBVR methods is based on the analysis of video shots [Chang et al. 1998; Deng and Manjunath 1998; Jain et al. 1999; Naphade and Huang 2001; Smoliar and Zhang 1994]. Shot boundaries are automatically detected by finding transitions (e.g., cut and fading) in video sequences. Each shot is then summarized as several key frames or clusters. For example, Jain et al. [1999] simply selected the first, middle, and last frames as key frames; Liu et al. [2003] extracted key frames by finding the peaks of the motion energy in the video sequence; Cheung and Zakhor [2003] proposed using the Voronoi video similarity for key frame selection; and Shen et al. [2005] modeled a video shot as a cluster described by the position, radius, and density of a hypersphere. Given a query clip, these methods search in the dataset by matching the shot key frames or clusters.

Existing CBVR methods do not consider video transformation because their ultimate goal is to find videos that are "semantically" similar, rather than "perceptually" similar, to the query clip. Moreover, they are regarded as typical of the partitioning and indexing approach, and suffer from the same problems mentioned in the previous section.

Searching by a sliding window is a popular approach [Chiu et al. 2008, Hampapur and Bolle 2001; Hua et al. 2004; Hoad and Zobel 2006; Kashino et al. 2003; Kim and Vasudev 2005]. Although this approach is simple and effective, the computation can be slow when scanning a long video sequence. However, if the indexing feature is represented in a histogram-based form, many frames can be skipped by exploiting the histogram pruning algorithm without influencing the matching result [Kashino et al. 2003]. In this paper, we develop a video copy detection method based on the window sliding approach.

The ordinal measure has been widely used in video copy detection [Chiu et al. 2008; Hampapur and Bolle 2001; Hua et al. 2004; Kim and Vasudev 2005; Yuan et al. 2004]. To extract the ordinal measure, each video frame is partitioned into $N_{x} \times N_{y}$ nonoverlapping blocks and the average intensity of each block is computed. Then, the blocks are ranked according to their average intensities; the ranking order is known as the frame's ordinal measure. The merit of the ordinal measure is its compact representation, as only a 9-dimensional vector is needed for a $3 \times 3$-block frame. Moreover, the measure is less sensitive to several video transformation types than general low-level image features (e.g., color histograms and texture descriptors). In addition to the ordinal measure, Hoad and Zobel [2006] proposed a compact video representation composed of color-shift and centroid-based signatures. The former computes the change of color histogram distribu- 
tions, while the latter computes the spatial movement of the lightest and darkest pixels. Consequently, each frame is represented by a 2-dimensional vector, which is more compact and robust than the ordinal measure.

Both the ordinal measure and the color-shift/centroid-based signature are extracted based on the whole frame region. We refer to them as "global descriptors," which have shown their robustness to whole region-preserved spatial transformation in many studies. However, if the frame is modified by partial region-discarded spatial transformation, its global descriptors might be totally different from those of the source frame.

Due to the limited capability of the global descriptors, various local descriptors have been investigated by many researchers as an alternative feature representation for image/video copy detection, e.g., the Harris descriptor [Schmid 1997], Scale-Invariant Feature Transform (SIFT) [Lowe 2004], local fingerprinting [Massoudi et al. 2006], and spatio-temporal features [DeMenthon and Doermann 2006; Willems et al. 2008]. In addition, the bag-of-words model is frequently applied to aggregate local descriptors of clusters for the sake of compact representation [Chiu et al. 2007; Poullot et al. 2008]. The comparative study of Law-To et al. [2007] shows that local descriptors are more robust than global descriptors in handling several video transformation types.

In this study, however, we find that global descriptors are better for whole regionpreserved spatial transformation, while local descriptors are better for partial regiondiscarded spatial transformation. These two types of descriptors can complement each other; therefore, combining them as a single feature representation improves the accuracy of detecting various video copies. This idea is different from the method of Wu et al. [2007], which employs the color histogram as the global descriptor for fast rejection at the coarse stage and the PCA-SIFT descriptor as the local descriptor for detailed matching at the fine stage. Since the global and local descriptors are applied separately at each stage, copies derived from partial region-discarded spatial transformation might be falsely rejected at the coarse stage.

Consider two video sequences that have the same content but different speeds: one is normal speed, and the other is double speed, as shown in Figs. 2(a) and 2(d), respectively. Since their contents do not synchronize, a window sliding-based method might obtain a lower similarity score and lead to a false negative. To remedy this problem, some methods have been proposed to take both spatial and temporal information into consideration. Hua et al. [2004], Hoad and Zobel [2006], and Chiu et al. [2008] applied the dynamic programming algorithm to solve the content synchronization problem; Joly et al. [2007] used a RANdom SAmple Consensus (RANSAC) algorithm to iteratively estimate the 
affine transformation parameters (say, resize, rotation, translation, and speed) between two video sequences; Law-To et al. [2006] proposed a voting algorithm for spatial and temporal registrations; and Kim et al. [2008] presented a non-crossing bipartite matching scheme to model the video linkage relation. A common point of these methods is that they assume the frame order of the video copy is not altered. They cannot handle frame order-changed temporal transformation, like the case shown in Fig. 2(e). To address this problem, we propose a novel spatio-temporal matching scheme by applying the Hough transform algorithm to search for the specific copy patterns. Our matching scheme can effectively deal with both spatial and temporal transformation categories mentioned in the previous section.

In order to efficiently search for video copies in a high-dimensional space, we propose using a popular algorithm for nearest neighbor search, namely, Locality-Sensitive Hashing (LSH). LSH uses several hash functions to hash an input item. An effective LSH function ensures that the hash collision probability of two items is proportional to their similarity. Several LSH functions have been derived based on the following distance and similarity functions, including Hamming distance, $L_{s}$ norms, $L_{2}$ distance on a sphere, arccos, and Jaccard coefficient [Andoni and Indyk 2008]. The LSH family has been successfully applied in several research fields, such as web mining [Das et al. 2007], bioinformatics [Buhler 2001], and image copy detection [Ke et al. 2004]. In this paper, we study the potential of the Jaccard coefficient-based LSH, i.e., min-hashing, in video copy detection.

\section{THE PROPOSED METHOD}

We propose a novel means of detecting various video copies in a continuous video sequence. To make video copy detection more efficient and effective, we use the compact min-hashing signature to reduce the similarity computation cost, and apply the robust spatio-temporal matching scheme to deal with a variety of video transformation. Fig. 4 shows an overview of the proposed method. Given a query clip, we scan the target sequence with a sliding window whose length is equal to the number of query frames. In this stage, the similarity between the query clip and a windowed sequence is measured based on their min-hashing signatures derived from the histograms. The windowed sequence with the similarity score exceeding a predefined threshold is denoted as a candidate, which will be further analyzed by spatio-temporal matching to determine whether it is a copy. In addition, based on the similarity score, we can decide how many frames the 
sliding window can skip when scanning the target sequence. The whole process is iterated until the sliding window reaches the end of the target sequence.

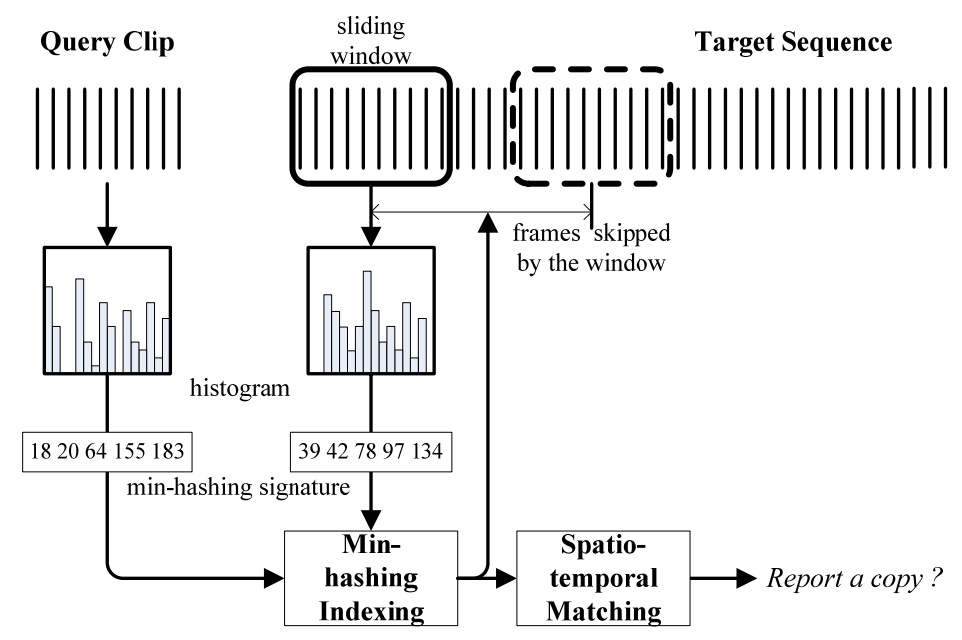

Fig. 4. An overview of the proposed method.

\subsection{Feature Representation and Similarity Measurement Prototype}

We use a histogram-based feature representation to model a video sequence. Histograms, which characterize the frequency distributions of feature vectors over a certain interval, have been widely employed in the multimedia field because of their effective discrimination and efficient computation properties [Swain and Ballard 1991; Ennesser and Medioni 1995]. Moreover, histograms do not count the frame order relation, so they are less sensitive to the temporal variations caused by frame order changes.

The histogram-based feature representation is formulated as follows. Let $Q=\left\{q_{i} \mid i=\right.$ $1,2, \ldots, n$ \} be a query clip, where $q_{i}$ is the $i$-th query frame. For each $q_{i}$, we extract its feature descriptors (e.g., the ordinal measure and SIFT descriptors) and quantize them to the corresponding clusters. By counting the number of feature descriptors in each cluster, we generate the histogram of frame $q_{i}$, denoted as $q H^{(i)}=\left\{q h_{1}^{(i)}, q h_{2}^{(i)}, \ldots, q h_{l}^{(i)}, \ldots, q h_{L}^{(i)}\right\}$, where $L$ is the number of clusters (histogram bins), and $q h_{l}^{(i)}$ is the number of feature descriptors classified into the $l$-th cluster. We then obtain the histogram of $Q$, denoted as $Q H=\left\{q h_{1}, q h_{2}, \ldots, q h_{l}, \ldots, q h_{L}\right\}$, by aggregating all frames' histograms, where

$$
q h_{l}=\sum_{i=1}^{n} q h_{l}^{(i)} \text {. }
$$

Given a target sequence $T=\left\{t_{j} \mid j=1,2, \ldots, m\right\}$, where $t_{j}$ is the $j$-th target frame, we repeat the above process to generate frame $t_{j}$ 's histogram. A window of length $n$ is em- 
ployed to slide over $T$. For a windowed sequence $C_{s}$ starting from frame $t_{s}$, i.e., $C_{s}=\left\{t_{j} \mid j\right.$ $\left.=s, s^{+} 1, \ldots, s^{+} n-1\right\}$, its histogram is denoted as $C H^{(s)}=\left\{c h_{1}^{(s)}, c h_{2}^{(s)}, \ldots, c h_{l}^{(s)}, \ldots, c h_{L}^{(s)}\right\}$. The similarity between $Q$ and $C_{s}$ is then computed by the Jaccard coefficient:

$$
J\left(Q, C_{s}\right)=\frac{\left|Q \cap C_{s}\right|}{\left|Q \cup C_{s}\right|}=\frac{\sum_{l=1}^{L} \min \left(q h_{l}, c h_{l}^{(s)}\right)}{\sum_{l=1}^{L} \max \left(q h_{l}, c h_{l}^{(s)}\right)} .
$$

where $|A|$ denotes the cardinality of set $A$. If $J\left(Q, C_{s}\right) \geq \theta_{\mathbf{J S}}$, where $\theta_{\mathrm{JS}}$ is a predefined threshold, we regard $C_{s}$ as a candidate that will be checked by spatio-temporal matching later.

Since the window length is determined when a query is given, histogram construction is an online process. The computation time required to construct $C H^{(s)}$ by summing $n$ histograms of $L$ dimensions is $\mathrm{O}(n L)$; and the computation time required to calculate the Jaccard coefficient is $\mathrm{O}(L)$. Therefore, the total computation time is $\mathrm{O}((n+1) L)$. In the following subsections, we present a compact feature representation, called the minhashing signature, to reduce the computation cost.

\subsection{Min-hashing Indexing}

Min-hashing, a locality sensitive hashing function derived based on the Jaccard coefficient, was proposed by Cohen et al. [2000] to solve the nearest neighbor search problem efficiently. The probability of two feature vectors having the same min-hashing signature is proportional to their similarity. In this study, we take the indices of the first $k$ non-zero elements of an $L$-dimensional feature vector as the hash values, where $1 \leq k<L$, and represent the feature vector by a $k$-dimensional min-hashing signature vector. As $k$ is smaller than the original feature dimension, the computation time for similarity measurement can be reduced.

To make the hashing process efficient, we employ the histogram-based feature representation described in Section 4.1 and directly use the index of the histogram bin as the hash value. Let $\Omega$ be the set of indices of histogram bins with nonzero values, i.e., $\Omega=\{l$ $\left.\mid h_{l}>0\right\}$. The elements in $\Omega$ are ranked in an ascending-order sequence $l(1), l(2), \ldots l(p), \ldots$ $l(|\Omega|)$, and $l(p) \in \Omega$ is the $p$-th smallest index in $\Omega$. A $k$-min-hashing signature SIG is defined as a sequence whose length is not larger than $k$ :

$$
S I G=\{l(p), p=1,2, \ldots, \min (|\Omega|, k)\} .
$$

In an ideal case, every histogram would be generated with an equal probability, and the cardinality of $\Omega$ would be much smaller than $L$; so that the proposed hashing would act approximately randomly. 
For query clip $Q$ and windowed sequence $C_{s}$, we denote their min-hashing signatures as $S I G_{Q}$ and $S I G_{C_{s}}$ respectively. Based on the min-hashing representation, the similarity between $Q$ and $C_{s}$ can be estimated by the following expression [Cohen et al. 2000]:

$$
M\left(Q, C_{s}\right)=\frac{\left|S I G_{Q} \cap S I G_{C_{s}}\right|}{\min \left(k,\left|S I G_{Q} \cup S I G_{C_{s}}\right|\right)} .
$$

Cohen et al. showed that the min-hashing similarity $M$ is proportional to the Jaccard similarity $J$ with a certain probability, which would be close to 1 for a suitably large choice of $k$. Hence, the candidate selection criterion can be modified as follows: if $M\left(Q, C_{s}\right) \geq \theta_{\mathrm{MS}}$ $=\delta \cdot \theta_{\mathrm{JS}}$, where $\delta$ is a real number between 0 and $1, C_{s}$ is regarded as a candidate for later spatio-temporal matching.

The computation time of the proposed min-hashing technique is still $\mathrm{O}(n L)$ due to the time spent on histogram construction for a query/windowed sequence. Therefore, we introduce a fast approximation approach to extract the min-hashing signature without histogram construction. For each query/target frame, we maintain a frame signature sig with maximal $g$ min-hashing values $(g<k)$ :

$$
\operatorname{sig}=\{l(p), p=1,2, \ldots, \min (|\Psi|, g)\},
$$

where $\Psi$, like $\Omega$, contains indices of histogram bins with nonzero values in ascending order. Given an $n$-frame sequence, we aggregate and sort its $n$ frame signatures to obtain an approximation $\Omega^{*}$ of $\Omega$. Consequently, we obtain an approximate min-hashing signature $S I G^{*}$ as

$$
S I G^{*}=\left\{l(p), p=1,2, \ldots, \min \left(\left|\Omega^{*}\right|, k\right)\right\} .
$$

The computation time required to generate $S I G^{*}$ for a query/windowed sequence is about $\mathrm{O}(n g \cdot \lg k)$.

Therefore, the total time spent on computing the min-hashing similarity between two sequences would be $\mathrm{O}(n g \cdot \lg k+k)$. This is comprised of $\mathrm{O}(n g \cdot \lg k)$ for generating the sequence signature and $\mathrm{O}(k)$ for calculating the intersection and union between two sequence signatures ${ }^{1}$. Compared with the computation of the Jaccard similarity, whose time complexity is $\mathrm{O}(n L)$, the min-hashing similarity can be computed more efficiently when $L$ is much larger than $g$.

Furthermore, in our implementation, the similarity in Equation (4) is simplified by replacing " $\min \left(k,\left|S I G_{Q} \cup S I G_{C_{s}}\right|\right) "$ in the denominator with a constant as follows:

\footnotetext{
${ }^{1}$ Since the elements in the signature are maintained in ascending order, the time required to compute the signature intersection and union is $\mathrm{O}(k)$.
} 


$$
M^{*}\left(Q, C_{s}\right)=\frac{\left|S I G_{Q}^{*} \cap S I G_{C_{s}}^{*}\right|}{k} .
$$

The computation cost of Equation (7) is expected to be approximately half that of Equation (4). Empirical studies show that the approximation works almost as well as the original form.

To summarize, we propose representing a video sequence by the min-hashing signature of the histogram feature. Some approximation forms are derived to speed up the signature generation and similarity measurement. The compact representation of the minhashing signature ensures that the video sequences can be matched efficiently.

4.2.1 Histogram Pruning for Speed-up. Even though the calculation of the minhashing signature is efficient, frame-by-frame scanning by the sliding window is impractical in terms of computation time. Thus, we adopt the histogram pruning algorithm proposed by Kashino et al. [2003] to accelerate the window sliding approach. The basic idea is to jump across frames during sliding, but still obtain the full-sliding result. The number of skipped frames is determined by the maximum increment of the sliding window, as well as the difference between the current window similarity and the predefined threshold $\theta_{\mathrm{MS}}$.

To incorporate this idea in our method, it is necessary to derive the maximum increment of the sliding window for the proposed min-hashing indexing. This is done as follows. Suppose we shift the window forward one frame in $T$. Frame $t_{s}$ slides out of the window and frame $t_{s+n}$ slides into the window, where $n$ is the sliding window length. In other words, $s i g_{s+n}$ is used instead of $s i g_{s}$ to construct $S I G_{(s+1)}^{*}$. It is clear that, according to Equation (7), the maximum increment from $M^{*}\left(Q, C_{s}\right)$ to $M^{*}\left(Q, C_{s+1}\right)$ is dependent on the maximum cardinality of the new incoming frame signature $s i g_{s+n}$, i.e., $\frac{\left|s g_{s+n}\right|}{k}$; thus, the maximum increment is $\frac{g}{k}$. Based on this observation, the number of skipped frames, $w$, can be calculated by:

$$
w= \begin{cases}\left\lfloor\frac{k}{g}\left(\theta_{\mathrm{MS}}-M^{*}\left(Q, C_{s}\right)\right)\right\rfloor+1, & \text { if } M^{*}\left(Q, C_{s}\right)<\theta_{\mathrm{MS}}, \\ 1, & \text { otherwise, }\end{cases}
$$

where $\lfloor x\rfloor$ rounds $x$ to the nearest integer less than or equal to $x$. It is guaranteed that no subsequence whose min-hashing similarity $M^{*}$ is greater than $\theta_{\mathrm{Ms}}$ will be missed in the sliding process, even if we skip $w$ frames. 
Note that $\theta_{\text {Ms }}$ plays an important role in determining the detection accuracy and the computation speed. A higher threshold will cause histogram pruning to skip a larger number of frames, and thus induce a higher precision rate and a lower recall rate in video copy detection.

\subsection{Spatio-temporal Matching}

Although the histogram-based feature representation is not affected by changing the frame order in a video sequence, it could be a drawback that the time relation between frames is not reflected. Some candidates found by the procedure described in Section 4.2 may be false positives; i.e., they may not be real copies of the query clip. On the other hand, continuously high similarity scores between frame pairs of two sequences strengthen their copy relation. Therefore, the similarity measurement should also consider the information about the temporal aspect in video copy detection.

We now present spatio-temporal matching. It serves as a verification process that further dissects the relation between the query and each candidate. The scheme compiles the spatial and temporal information of all the frame pairs of two video sequences, and it can be visualized through a 2D intensity map. A computer vision-based algorithm is then applied to search the map for the specific copy patterns.

To simplify the notation, a candidate sequence is denoted as $C=\left\{c_{p} \mid p=1,2, \ldots, n\right\}$, where $c_{p}$ is the $p$-th frame of $C$. The histogram of frame $c_{p}$ is re-written as $c H^{(p)}=\left\{c h_{1}^{(p)}, c h_{2}^{(p)}, \ldots, c h_{l}^{(p)}, \ldots, c h_{L}^{(p)}\right\}$. We define the pairwise matrix for $Q$ and $C$, which is an $n \times n$ matrix representing all-pair frame similarities, as follows:

$$
\mathbf{P M}(Q, C)=\left\{m_{i p}, i=1 \ldots n, p=1 \ldots n \mid m_{i p}=\sum_{l=1}^{L} \min \left(q h_{l}^{(i)}, c h_{l}^{(p)}\right)\right\} .
$$

The (i,p)-th element $m_{i p} \in[0,1]$ stores the frame similarity between the $i$-th query frame and the $p$-th candidate frame, which is the intersection of the two frames' histograms.

The pairwise matrix PM can be visualized by plotting its frame similarities as graylevel intensities. To illustrate this point, Fig. 5 shows four cases: (a) $\mathbf{P M}\left(Q, C_{1}\right)$; (b) $\mathbf{P M}\left(Q, C_{2}\right)$; (c) $\mathbf{P M}\left(Q, C_{3}\right)$; and (d) $\mathbf{P M}\left(Q, C_{4}\right)$. The $\mathrm{X}$-axis and the $\mathrm{Y}$-axis indicate the candidate frame index and the query frame index, respectively. Except for $C_{1}$, the other three candidates are copies of $Q: C_{2}$ enhances the brightness of $Q, C_{3}$ is a slow motion copy, and $C_{4}$ swaps $Q$ 's first-half subsequence and second-half subsequence. We observe that the intensity distribution in Fig. 5(a) is very scattered, whereas slant line patterns appear clearly in Figs. 5(b)-(d). The slant line patterns with different orientations and 
positions also reflect the types of temporal transformation that were applied to the source video.

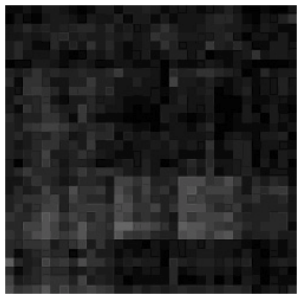

(a)

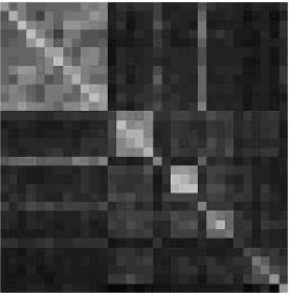

(b)

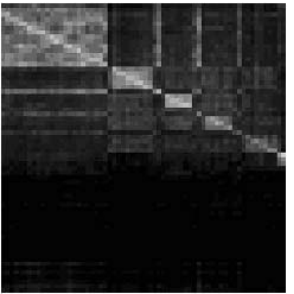

(c)

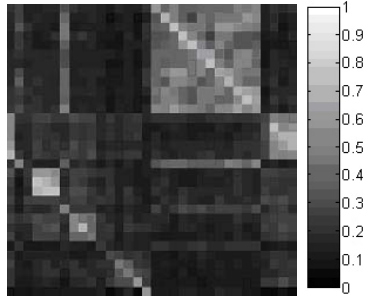

(d)

Fig. 5. The pairwise matrixes: (a) $\mathbf{P M}\left(Q, C_{1}\right)$; (b) $\mathbf{P M}\left(Q, C_{2}\right)$; (c) $\mathbf{P M}\left(Q, C_{3}\right)$; and (d) $\mathbf{P M}\left(Q, C_{4}\right)$. Except for $C_{1}$, the other candidates are copies of $Q$, and their corresponding matrixes exhibit slant line patterns clearly.

The slant line pattern, which manifests a set of consecutive frame pairs with high similarity scores, indicates a possible copy relation at that interval. Based on this observation, the task of spatio-temporal matching involves detecting slant line patterns on the pairwise matrix. Since there may be multiple slant lines with various positions and orientations, the detection process should be able to find any kind of patterns. To this end, we use the Hough transform [Sonka et al. 1999], a well-known algorithm for detecting objects in an image.

The detection process is implemented as follows. First, we use the Sobel edge detection method on the pairwise matrix PM to determine the magnitude and direction of each pixel. The pixels are considered as potential line points if they satisfy the following two conditions: (1) the pixel magnitudes exceed a given threshold $\theta_{\mathbf{E M}}$, and (2) the pixel directions are within a certain range $\Theta_{\text {ED }}$. These potential line points are then projected to the Hough space. Finally, local maxima in the Hough space that are greater than a threshold $\theta_{\mathbf{L M}}$ are regarded as slant lines on PM.

While $\theta_{\mathbf{E M}}$ is usually decided empirically, $\Theta_{\mathbf{E D}}$ can be determined by the video speed range $\Theta_{\mathbf{E D}}=\left[\arctan \left(\rho_{L}\right), \arctan \left(\rho_{H}\right)\right]$, where $\rho_{L}$ and $\rho_{H}$ are, respectively, the lowest and highest video speeds to be considered. This is because the speed range also constrains the range of the slant line orientation on PM. For example, to detect video copies with the speed range between $0.5 \times$ and $2 \times$, we can set $\Theta_{\mathbf{E D}}=\left[26.57^{\circ}-\varepsilon, 63.43^{\circ}+\varepsilon\right]$, where $\varepsilon$ is a small tolerance value. $\theta_{\mathbf{L M}}$ can be decided by a proportion of $n$.

Fig. 6 shows two examples of detecting slant line patterns. Figs. 6(a)-(d) illustrate the results for the case in Fig. 5(c): (a) is the pairwise matrix; (b) shows the edge points on PM that satisfy the threshold conditions of $\theta_{\mathbf{E M}}$ and $\Theta_{\mathbf{E D}}$; (c) is the Hough space, where 
the rectangle labels the location of the local maximum; and (d) shows the detection result with a dashed line. Figs. 6(e)-(h) illustrate the results for the case in Fig. 5(d). The two examples demonstrate that the proposed matching scheme can detect multiple slant lines in various positions and orientations. They also demonstrate the proposed scheme yields excellent performance in handling temporal transformation.

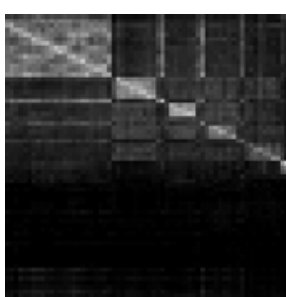

(a)

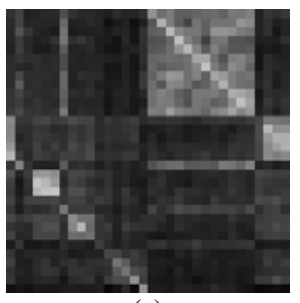

(e)

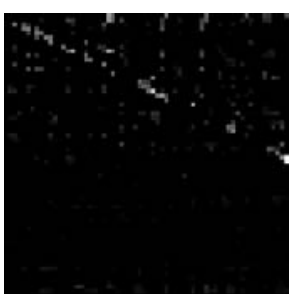

(b)

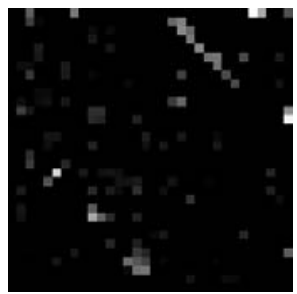

(f)

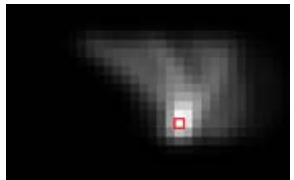

(c)

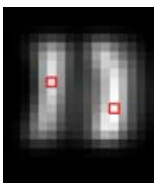

(g)

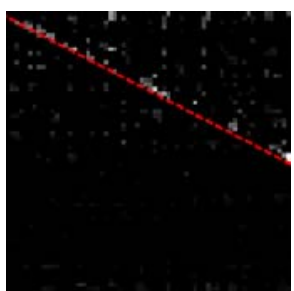

(d)

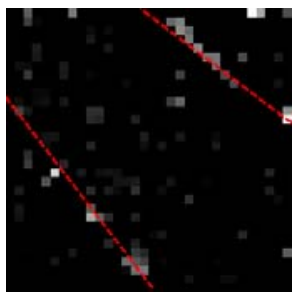

(h)

Fig. 6. (a)-(d) illustrate the slant line pattern detection process for $\mathbf{P M}\left(Q, C_{3}\right)$ : (a) the pairwise matrix; (b) the edge points on PM; (c) the Hough space, where the rectangle labels the location of the local maximum; and (d) the detection result with a dashed line; (e)-(h) illustrate the detection results for $\mathbf{P M}\left(Q, C_{4}\right)$.

4.3.1 Inverted Indexing for Speed-up. A naive way to construct the pairwise matrix PM is to compute all-pair frame similarities according to Equation (9). The time complexity is $\mathrm{O}\left(L \cdot n^{2}\right)$. However, $\mathbf{P M}$ is a sparse matrix that can be constructed by counting only a few frame pairs. Here we employ an inverted indexing technique to reduce the computation cost of constructing PM.

Let IT be the inverted table that contains $L$ cells to store video frames. For every query frame $q_{i}$, if its $l$-th histogram bin $q h_{l}^{(i)}>0, q_{i}$ is inserted into the $l$-th cell of IT. We apply the same step to insert every candidate frame $c_{p}$ into the corresponding cells of IT. Then, we construct PM by scanning IT as follows: for each frame pair $\left(q_{i}, c_{p}\right)$ found in the $l$-th cell of $\mathbf{I T}$, we update the $(i, p)$-th element $m_{i p}$ of $\mathbf{P M}$ by adding $\min \left(q h_{l}^{(i)}, c h_{l}^{(p)}\right)$.

When only a few frame pairs intersect in some histogram bins, using the inverted table can reduce the computation cost substantially. The computation time mainly comprises of inserting frames into IT and accumulating the number of frame pairs in IT. Further discussions are given in experiments. 


\section{EXPERIMENTS}

To evaluate the proposed method, we compiled a video dataset for use in several experiments. The code was implemented in $\mathrm{C}++^{+}$, and the evaluation was carried out on a PC with a $1.8 \mathrm{GHz} \mathrm{CPU}$ and 2GB RAM.

\subsection{Video Dataset}

A 6.1-hour video sequence was compiled from the MPEG-7 video CD collection and the Open Video Project (http://www.open-video.org/). Its contents included sports programs, news broadcasts, and documentaries. We transformed these video data into the following uniform format: MPEG-1, 320×240 pixels, and 30 frames per second (fps). This dataset served as the target sequence.

From the target sequence, we randomly extracted 31 subsequences, each of 30 seconds duration. Each subsequence derived twelve video copies by applying the common video transformation types listed in Table 1. Fig. 7 gives two examples of frame orderchanged temporal transformation used in the experiments. There were totally $372(31 \times 12)$ video copies, which served as the query clips. Each copy was used to detect the corresponding subsequence in the target sequence. Note that the definition of the target sequence and query clips here is different from that given in Section 2. We made this modification to fit the experiment's purpose; that is, to only generate a small size of the query dataset.

Since a continuous video sequence contains many identical or near-duplicate frames, it is not necessary to use every frame in the sequence for matching. Therefore, we selected a key frame every 15 frames of the target sequence. In other words, the frame rate of the target sequence became $2 \mathrm{fps}$. In addition, before starting the detection process, we had to determine the frame rate of the query clip, which is commonly available from the file header. The query clip was re-sampled so that its frame rate is synchronized with that of the target sequence. For example, a 30-second query clip with 30fps became a 60frame sequence after re-sampling.

Table 1. Different types of video transformation.

\begin{tabular}{|l|l|l|}
\hline Category & Type & Description \\
\hline Whole region-preserved & Brightness & Enhance the brightness by 20\%. \\
\cline { 2 - 3 } spatial transformation & Compression & Set the compression quality 50\% (by IndeoR 5.10). \\
\cline { 2 - 3 } & Noise & Add 10\% random noise. \\
\hline
\end{tabular}




\begin{tabular}{|c|c|c|}
\hline & Equalization & Equalize the color histogram. \\
\hline & Resolution change & Change the frame resolution to $120 \times 90$ pixels. \\
\hline \multirow{2}{*}{$\begin{array}{l}\text { Partial region-discarded } \\
\text { spatial transformation }\end{array}$} & Cropping & Crop the top and bottom frame regions by $10 \%$ each. \\
\hline & Zooming in & Zoom in to the frame by $10 \%$. \\
\hline \multirow{3}{*}{$\begin{array}{l}\text { Frame number-changed } \\
\text { temporal transformation }\end{array}$} & Slow motion & Halve the video speed. \\
\hline & Fast forward & Double the video speed. \\
\hline & Frame rate change & Change the frame rate to $15 \mathrm{fps}$. \\
\hline \multirow{2}{*}{$\begin{array}{l}\text { Frame order-changed } \\
\text { temporal transformation }\end{array}$} & Swap & Swap the first-half subsequence and the second-half one. \\
\hline & Insertion/deletion & Delete middle $50 \%$ of frames and insert unrelated frames. \\
\hline
\end{tabular}

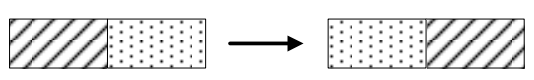

(a)

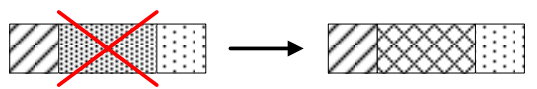

(b)

Fig. 7. Examples of frame order-changed temporal transformation used in the experiments: (a) Swap the firsthalf subsequence and the second-half one. (b) Delete middle $50 \%$ of frames and insert unrelated frames.

\subsection{Feature Extraction}

From each frame, we extracted the ordinal measure and the SIFT descriptor to serve as the global descriptor and local descriptor, respectively. To extract the ordinal measure, we partitioned each video frame into $N_{x} \times N_{y}$ non-overlapping blocks and computed their intensity ranks. The rank order is known as the ordinal measure of the frame. Since there were $\left(N_{x} \times N_{y}\right)$ ! possible permutations of the ordinal measure, we used a histogram with $\left(N_{x} \times N_{y}\right)$ ! bins to represent a video sequence, where each frame was assigned to a histogram bin according to its ordinal measure. Here $N_{x}=3$ and $N_{y}=2$.

To extract SIFT descriptors of each frame, we located the local extrema in the DOG (Differential-of-Gaussian) scale space and computed their orientation histograms in the image space. We used a training dataset collected from another video collection, and applied the LBG (Linde-Buzo-Gray) algorithm [Sayood 1996] to generate a codebook of $L$ codewords. Then, each SIFT descriptor was quantized to the nearest codeword and assigned to the corresponding histogram bin. Subsequently, a video sequence was represented by a histogram with $L$ bins. Here $L=1024$. For every frame, we extracted one ordinal measure and averagely 22.84 SIFT descriptors in this dataset.

As mentioned earlier, the ordinal measure and the SIFT descriptor can be combined to improve the accuracy. To this end, we simply executed the search twice using the ordinal measure and the SIFT descriptor individually to collect their corresponding candidate sets, which were then merged into a single one. For a candidate sequence in the merged set, 
let $\mathbf{P M}_{\text {ordinal }}$ and $\mathbf{P} \mathbf{M}_{\mathrm{SIFT}}$ be the pairwise matrixes generated based on ordinal measure and SIFT descriptor, respectively; and let $\mathbf{P} \mathbf{M}_{\text {combination }}$ be the combined form written as:

$\mathbf{P M}_{\text {combination }}=\alpha \cdot \mathbf{P M}_{\text {ordinal }}+(1-\alpha) \cdot \mathbf{P} \mathbf{M}_{\mathrm{SIFT}}$,

where $\alpha \in[0,1]$ is a weighting factor that controls the relative importance of $\mathbf{P M}_{\text {ordinal }}$ and $\mathbf{P M}_{\mathrm{SIFT}}$. We set $\alpha=0.5$ throughout the experiments.

\subsection{Evaluation Metric}

We used the following detection criteria for the accuracy evaluation. A detection result was considered correct if it had any overlap with the region from which the query was extracted. The recall and precision rates were used to evaluate the accuracy of the detection result:

$$
\begin{aligned}
& \text { recall }=T P /(T P+F N), \\
& \text { precision }=T P /(T P+F P),
\end{aligned}
$$

where True Positives (TP) refer to positive examples correctly labeled as positives; False Negatives (FN) refer to positive examples incorrectly labeled as negatives; and False Positives (FP) refer to negative examples incorrectly labeled as positives. We also used the F-measure, calculated as:

$$
\text { F-measure }=(2 \times \text { recall } \times \text { precision }) /(\text { recall }+ \text { precision }) \text {. }
$$

\subsection{Overview of Methods Evaluated}

We implemented the following seven methods for performance evaluation:

(1) The ordinal measure with spatio-temporal matching (abbreviated as "OM+STM").

(2) The SIFT descriptor with spatio-temporal matching ("SD+STM").

(3) The combination of Methods (1) and (2) using Equation (10) ("OM+SD+STM").

(4) The ordinal measure without spatio-temporal matching ("OM").

(5) The SIFT descriptor without spatio-temporal matching ("SD").

(6) Method (2) without min-hashing ("SD+STM-MH").

(7) Hoad and Zobel's method [2006] ("HZ").

Methods (1) and (2) used different min-hashing features with spatio-temporal matching, and their combination yielded Method (3). We implemented Methods (4) and (5) to see the effect without the proposed spatio-temporal matching scheme. Method (6) was implemented to assess the proposed min-hashing indexing. The threshold parameters used in Methods (1)-(5) were configured as follows. For min-hashing similarity computation, we set $\theta_{\mathbf{M S}}=0.5$; for spatio-temporal matching, we set $\theta_{\mathbf{E M}}=G / 4$, where $G$ is the 
maximum among all edge magnitudes in the pairwise matrix, $\Theta_{\mathbf{E D}}=\left[20^{\circ}, 70^{\circ}\right]$, and $\theta_{\mathbf{L M}}$ $=n / 5$. In Method (6), the Jaccard similarity threshold $\theta_{\mathrm{JS}}$ was adjusted to control the trade-off between the recall and precision rates. We finally set $\theta_{\mathrm{JS}}=0.35$ since its accuracy was closest to that of Method (2) in several transformation types. We can thus compare the computation cost of Method (2) and Method (6) (with min-hashing indexing vs. without min-hashing indexing). In Method (7), the recall and precision rates were measured after $x$ results were detected, where $x$ is the number of positives (video copies) in the target sequence.

Recall that $k$ and $g$ are the min-hashing signature lengths of a sequence and a frame, respectively. The configurations of the $(k, g)$ pairs were set according to the feature used. For the methods using the SIFT descriptor, i.e., Methods (2) and (5), we used the following ten empirical $(k, g)$ pairs in the experiments: $(10,2),(20,3),(30,3),(40,3),(50,4)$, $(60,4),(70,5),(80,5),(90,6)$, and $(100,6)$. For the methods using the ordinal measure, i.e., Methods (1) and (4), each frame had only one ordinal measure; hence, $g$ was always set to 1. In Method (3), which combines the ordinal measure and SIFT descriptor, we used the above ten $(k, g)$ pairs for the SIFT descriptor part and the pair $(k, g)=(30,1)$ for the ordinal measure part. Methods (6) and (7) did not involve $k$ and $g$.

Hoad and Zobel's method, i.e., Method (7), is one of the state of the art methods that use the window sliding approach. We implemented their method as follows. In each frame, the color-shift signature was extracted by using 16 bins for each of the three color channels in YCbCr. The Manhattan distance was used to calculate the histogram distance of two adjacent frames. To extract the centroid-based signature, we identified the lightest and darkest $5 \%$ of pixels in each frame, and computed their average coordinates as the centroid location. We calculated the Euclidean distance of the centroids between two adjacent frames. The two distance signatures were combined into a single vector to represent a frame, and approximate string matching was applied for similarity measurement. This method can provide a perspective on the typical window sliding approach using a global descriptor.

\subsection{Detection Accuracy}

In the following subsections, we show and discuss the accuracy of the compared methods in terms of spatial and temporal transformation.

5.5.1 Whole region-preserved spatial transformation. This category includes brightness enhancement, compression, noise addition, histogram equalization, and frame reso- 
lution change, which have been widely tested by existing methods. The results are listed in Tables 2-6. The bold font indicates the highest F-measure scores in the table.

The length of the min-hashing signature has quite a large impact on the retrieval accuracy of the ordinal-based methods (i.e., Methods (1) and (4)). The recall rate degrades and the precision rate improves as $k$ grows to 60 . Since the query clip contains 60 frames after re-sampling, the maximum length of the ordinal-based min-hashing signature is 60 . This explains why the performance will not change when $k>60$. In contrast, the length of the min-hashing signature has a limited impact in the SIFT-based methods (i.e., Methods (2) and (5)), with slight variations in the recall and precision rates under various $k$.

The performance of the SIFT-based methods is superior to that of the ordinal-based methods for the brightness, compression, and noise transformation types. However, for the equalization and resolution change types, the recall rates of the SIFT-based methods decline sharply. This is because applying the two transformation types might alter the original SIFT descriptor's property substantially. Compared with the SIFT-based methods, the ordinal-based methods yield nearly similar results for all types of whole regionpreserved spatial transformation. With a suitable choice of $k$, the ordinal-based methods would gain a more robust performance than the SIFT-based methods in this transformation category.

The proposed spatio-temporal matching scheme is effective in improving the precision rates of both ordinal-based and SIFT-based methods. In particular for the SIFTbased methods, Method (2) obtains a substantial improvement from Method (5). From another perspective, Method (5) retrieves a large number of false positives, while Method (2) utilizes the proposed matching scheme to remove these false positives effectively.

Method (6) obtains extremely good results for the brightness, compression, and noise transformation types. Its accuracy served as an upper bound for Method (2) in these transformation types. From Tables 2-6, we observe that the gap is not very significant.

Hoad and Zobel's method, i.e., Method (7), performs well for the compression, noise, and resolution change types, but not as well for the brightness and equalization types. This is because its color-shift signature counts the histograms of the color channels that might vary widely after applying brightness enhancement or histogram equalization.

Among all the methods, Method (3), i.e., the combination of Methods (1) and (2), yields the most robust results in all types of whole region-preserved spatial transformation. Method (3) exhibits a similar accuracy distribution to that of Method (2); however, because of the combination of the ordinal measure and SIFT descriptor, the accuracy distribution of Method (3) is more compact and expresses better precision and recall rates. 
Table 2. The precision and recall rates for brightness transformation.

\begin{tabular}{|c|c|c|c|c|c|c|c|c|c|c|c|}
\hline & & $k=10$ & $k=20$ & $k=30$ & $k=40$ & $k=50$ & $k=60$ & $k=70$ & $k=80$ & $k=90$ & $k=100$ \\
\hline \multirow{2}{*}{$\begin{array}{c}(1) \\
\text { OM+STM }\end{array}$} & $\mathbf{R}$ & 1.0000 & 0.9355 & 0.9355 & 0.7419 & 0.5806 & 0.2258 & 0.2258 & 0.2258 & 0.2258 & 0.2258 \\
\hline & $\mathbf{P}$ & 0.3523 & 0.6591 & 0.8529 & 1.0000 & 1.0000 & 1.0000 & 1.0000 & 1.0000 & 1.0000 & 1.0000 \\
\hline \multirow{2}{*}{$\begin{array}{c}(2) \\
\text { SD+STM }\end{array}$} & $\mathbf{R}$ & 0.9032 & 0.9677 & 0.9677 & 1.0000 & 0.9677 & 0.9677 & 0.9677 & 0.9355 & 0.9355 & 0.9355 \\
\hline & $\mathbf{P}$ & 0.9333 & 0.9375 & 0.9091 & 0.9394 & 0.9375 & 0.9677 & 0.9677 & 0.9677 & 0.9677 & 0.9677 \\
\hline \multirow{2}{*}{$\begin{array}{c}\text { (3) } \\
\mathrm{OM}+\mathrm{SD}+\mathrm{STM}\end{array}$} & $\mathbf{R}$ & 1.0000 & 1.0000 & 1.0000 & 1.0000 & 1.0000 & 1.0000 & 1.0000 & 1.0000 & 1.0000 & 1.0000 \\
\hline & $\mathbf{P}$ & 0.9394 & 0.9394 & 0.9118 & 0.9394 & 0.9394 & 0.9688 & 0.9688 & 0.9688 & 0.9688 & 0.9688 \\
\hline \multirow{2}{*}{$\begin{array}{l}(4) \\
\text { OM }\end{array}$} & $\mathbf{R}$ & 1.0000 & 0.9355 & 0.9355 & 0.7419 & 0.5806 & 0.2258 & 0.2258 & 0.2258 & 0.2258 & 0.2258 \\
\hline & $\mathbf{P}$ & 0.1348 & 0.5686 & 0.8056 & 0.9583 & 1.0000 & 1.0000 & 1.0000 & 1.0000 & 1.0000 & 1.0000 \\
\hline \multirow{2}{*}{$\begin{array}{l}\text { (5) } \\
\text { SD }\end{array}$} & $\mathbf{R}$ & 1.0000 & 0.9677 & 0.9677 & 1.0000 & 0.9677 & 0.9677 & 0.9677 & 0.9355 & 0.9355 & 0.9355 \\
\hline & $\mathbf{P}$ & 0.0110 & 0.0258 & 0.0296 & 0.0546 & 0.0571 & 0.0993 & 0.0962 & 0.1312 & 0.1394 & 0.1648 \\
\hline \multirow{2}{*}{$\begin{array}{c}(6) \\
\text { SD+STM-MH } \\
\end{array}$} & $\mathbf{R}$ & 1.0000 & 1.0000 & 1.0000 & 1.0000 & 1.0000 & 1.0000 & 1.0000 & 1.0000 & 1.0000 & 1.0000 \\
\hline & $\mathbf{P}$ & 1.0000 & 1.0000 & 1.0000 & 1.0000 & 1.0000 & 1.0000 & 1.0000 & 1.0000 & 1.0000 & 1.0000 \\
\hline \multirow{2}{*}{$\begin{array}{l}\text { (7) } \\
\text { HZ }\end{array}$} & $\mathbf{R}$ & 0.7742 & 0.7742 & 0.7742 & 0.7742 & 0.7742 & 0.7742 & 0.7742 & 0.7742 & 0.7742 & 0.7742 \\
\hline & $\mathbf{P}$ & 0.7742 & 0.7742 & 0.7742 & 0.7742 & 0.7742 & 0.7742 & 0.7742 & 0.7742 & 0.7742 & 0.7742 \\
\hline
\end{tabular}

Table 3. The precision and recall rates for compression transformation.

\begin{tabular}{|c|c|c|c|c|c|c|c|c|c|c|c|}
\hline & & $k=10$ & $k=20$ & $k=30$ & $k=40$ & $k=50$ & $k=60$ & $k=70$ & $k=80$ & $k=90$ & $k=100$ \\
\hline \multirow{2}{*}{$\begin{array}{c}(1) \\
\text { OM+STM } \\
\end{array}$} & $\mathbf{R}$ & 1.0000 & 0.9355 & 0.9355 & 0.7742 & 0.6129 & 0.2581 & 0.2581 & 0.2581 & 0.2581 & 0.2581 \\
\hline & $\mathbf{P}$ & 0.3483 & 0.6304 & 0.8529 & 0.9231 & 0.9500 & 1.0000 & 1.0000 & 1.0000 & 1.0000 & 1.0000 \\
\hline \multirow{2}{*}{$\begin{array}{c}(2) \\
\text { SD+STM }\end{array}$} & $\mathbf{R}$ & 0.8387 & 0.8710 & 0.9677 & 0.9677 & 0.9355 & 0.9355 & 0.9355 & 0.9355 & 0.8710 & 0.8710 \\
\hline & $\mathbf{P}$ & 0.9286 & 0.9310 & 0.9677 & 0.9677 & 0.9355 & 0.9667 & 0.9667 & 0.9667 & 0.9643 & 0.9643 \\
\hline \multirow{2}{*}{$\begin{array}{c}\text { (3) } \\
\mathrm{OM}+\mathrm{SD}+\mathrm{STM}\end{array}$} & $\mathbf{R}$ & 1.0000 & 0.9355 & 0.9677 & 0.9677 & 0.9677 & 0.9677 & 0.9677 & 1.0000 & 0.9677 & 0.9677 \\
\hline & $\mathbf{P}$ & 0.9118 & 0.9355 & 0.9677 & 0.9677 & 0.9677 & 0.9375 & 0.9677 & 0.9688 & 0.9677 & 0.9677 \\
\hline \multirow{2}{*}{$\begin{array}{l}(4) \\
\text { OM }\end{array}$} & $\mathbf{R}$ & 1.0000 & 0.9355 & 0.9355 & 0.7742 & 0.6129 & 0.2581 & 0.2581 & 0.2581 & 0.2581 & 0.2581 \\
\hline & $\mathbf{P}$ & 0.1360 & 0.5577 & 0.8056 & 0.9231 & 0.9500 & 1.0000 & 1.0000 & 1.0000 & 1.0000 & 1.0000 \\
\hline \multirow{2}{*}{$\begin{array}{l}(5) \\
\text { SD }\end{array}$} & $\mathbf{R}$ & 0.9032 & 0.9032 & 1.0000 & 1.0000 & 0.9667 & 0.9667 & 0.9667 & 0.9355 & 0.9032 & 0.9032 \\
\hline & $\mathbf{P}$ & 0.0103 & 0.0223 & 0.0262 & 0.0458 & 0.0439 & 0.0699 & 0.0699 & 0.0945 & 0.0930 & 0.1197 \\
\hline \multirow{2}{*}{$\begin{array}{c}\mathbf{( 6 )} \\
\text { SD+STM-MH }\end{array}$} & $\mathbf{R}$ & 0.9677 & 0.9677 & 0.9677 & 0.9677 & 0.9677 & 0.9677 & 0.9677 & 0.9677 & 0.9677 & 0.9677 \\
\hline & $\mathbf{P}$ & 1.0000 & 1.0000 & 1.0000 & 1.0000 & 1.0000 & 1.0000 & 1.0000 & 1.0000 & 1.0000 & 1.0000 \\
\hline \multirow{2}{*}{$\begin{array}{l}\text { (7) } \\
\text { HZ }\end{array}$} & $\mathbf{R}$ & 0.9355 & 0.9355 & 0.9355 & 0.9355 & 0.9355 & 0.9355 & 0.9355 & 0.9355 & 0.9355 & 0.9355 \\
\hline & $\mathbf{P}$ & 0.9355 & 0.9355 & 0.9355 & 0.9355 & 0.9355 & 0.9355 & 0.9355 & 0.9355 & 0.9355 & 0.9355 \\
\hline
\end{tabular}

Table 4. The precision and recall rates for noise transformation.

\begin{tabular}{|c|c|c|c|c|c|c|c|c|c|c|c|}
\hline & & $k=10$ & $k=20$ & $k=30$ & $k=40$ & $k=50$ & $k=60$ & $k=70$ & $k=80$ & $k=90$ & $k=100$ \\
\hline \multirow{2}{*}{$\begin{array}{c}(1) \\
\text { OM+STM }\end{array}$} & $\mathbf{R}$ & 1.0000 & 0.9355 & 0.9355 & 0.7419 & 0.5806 & 0.2258 & 0.2258 & 0.2258 & 0.2258 & 0.2258 \\
\hline & $\mathbf{P}$ & 0.3605 & 0.6905 & 0.9063 & 1.0000 & 1.0000 & 1.0000 & 1.0000 & 1.0000 & 1.0000 & 1.0000 \\
\hline \multirow{2}{*}{$\begin{array}{c}(2) \\
\text { SD+STM }\end{array}$} & $\mathbf{R}$ & 0.8065 & 0.8065 & 0.9355 & 0.9677 & 0.8710 & 0.9032 & 0.9032 & 0.8710 & 0.8387 & 0.8710 \\
\hline & $\mathbf{P}$ & 0.8929 & 0.8065 & 0.8286 & 0.8824 & 0.9310 & 0.9032 & 0.9032 & 0.9310 & 0.9286 & 0.9310 \\
\hline \multirow{2}{*}{$\begin{array}{c}\text { (3) } \\
\text { OM+SD+STM }\end{array}$} & $\mathbf{R}$ & 1.0000 & 1.0000 & 1.0000 & 1.0000 & 1.0000 & 1.0000 & 1.0000 & 1.0000 & 1.0000 & 1.0000 \\
\hline & $\mathbf{P}$ & 0.9118 & 0.8378 & 0.8378 & 0.8857 & 0.9394 & 0.9118 & 0.9118 & 0.9394 & 0.9394 & 0.9394 \\
\hline \multirow{2}{*}{$\begin{array}{l}\text { (4) } \\
\text { OM }\end{array}$} & $\mathbf{R}$ & 1.0000 & 0.9355 & 0.9355 & 0.7419 & 0.5806 & 0.2258 & 0.2258 & 0.2258 & 0.2258 & 0.2258 \\
\hline & $\mathbf{P}$ & 0.1396 & 0.6304 & 0.8529 & 0.9583 & 1.0000 & 1.0000 & 1.0000 & 1.0000 & 1.0000 & 1.0000 \\
\hline \multirow{2}{*}{$\begin{array}{l}\text { (5) } \\
\text { SD }\end{array}$} & $\mathbf{R}$ & 0.8387 & 0.8387 & 0.9355 & 0.9677 & 0.9355 & 0.9355 & 0.9355 & 0.9355 & 0.9032 & 0.9032 \\
\hline & $\mathbf{P}$ & 0.0089 & 0.0185 & 0.0221 & 0.0384 & 0.0389 & 0.0556 & 0.0577 & 0.0788 & 0.0824 & 0.1089 \\
\hline \multirow{2}{*}{$\begin{array}{c}\mathbf{( 6 )} \\
\text { SD+STM-MH }\end{array}$} & $\mathbf{R}$ & 1.0000 & 1.0000 & 1.0000 & 1.0000 & 1.0000 & 1.0000 & 1.0000 & 1.0000 & 1.0000 & 1.0000 \\
\hline & $\mathbf{P}$ & 1.0000 & 1.0000 & 1.0000 & 1.0000 & 1.0000 & 1.0000 & 1.0000 & 1.0000 & 1.0000 & 1.0000 \\
\hline \multirow{2}{*}{$\begin{array}{l}\text { (7) } \\
\text { HZ }\end{array}$} & $\mathbf{R}$ & 0.9032 & 0.9032 & 0.9032 & 0.9032 & 0.9032 & 0.9032 & 0.9032 & 0.9032 & 0.9032 & 0.9032 \\
\hline & $\mathbf{P}$ & 0.9032 & 0.9032 & 0.9032 & 0.9032 & 0.9032 & 0.9032 & 0.9032 & 0.9032 & 0.9032 & 0.9032 \\
\hline
\end{tabular}

Table 5. The precision and recall rates for equalization transformation.

\begin{tabular}{|c|c|c|c|c|c|c|c|c|c|c|c|}
\hline & & $k=10$ & $k=20$ & $k=30$ & $k=40$ & $k=50$ & $k=60$ & $k=70$ & $k=80$ & $k=90$ & $k=100$ \\
\hline \multirow{2}{*}{$\begin{array}{c}\text { (1) } \\
\text { OM+STM }\end{array}$} & $\mathbf{R}$ & 0.8387 & 0.9032 & 0.8387 & 0.4194 & 0.2581 & 0.0323 & 0.0323 & 0.0323 & 0.0323 & 0.0323 \\
\hline & $\mathbf{P}$ & 0.2766 & 0.6829 & 0.8667 & 1.0000 & 1.0000 & 1.0000 & 1.0000 & 1.0000 & 1.0000 & 1.0000 \\
\hline \multirow{2}{*}{$\begin{array}{c}(2) \\
\text { SD+STM }\end{array}$} & $\mathbf{R}$ & 0.2903 & 0.3548 & 0.3548 & 0.3226 & 0.2581 & 0.3226 & 0.2903 & 0.1935 & 0.1613 & 0.1613 \\
\hline & $\mathbf{P}$ & 0.6429 & 0.7333 & 0.7857 & 0.8333 & 1.0000 & 0.9091 & 0.9000 & 0.8571 & 0.8333 & 0.8333 \\
\hline \multirow{2}{*}{$\begin{array}{c}\text { (3) } \\
\mathrm{OM}+\mathrm{SD}+\mathrm{STM}\end{array}$} & $\mathbf{R}$ & 0.8065 & 0.8065 & 0.8065 & 0.7742 & 0.7419 & 0.7419 & 0.7419 & 0.7419 & 0.7419 & 0.7419 \\
\hline & $\mathbf{P}$ & 0.8333 & 0.8621 & 0.8929 & 0.9231 & 1.0000 & 0.9583 & 0.9583 & 0.9583 & 0.9583 & 0.9583 \\
\hline \multirow{2}{*}{$\begin{array}{l}\text { (4) } \\
\text { OM }\end{array}$} & $\mathbf{R}$ & 0.9355 & 0.9032 & 0.8387 & 0.4194 & 0.2581 & 0.0323 & 0.0323 & 0.0323 & 0.0323 & 0.0323 \\
\hline & $\mathbf{P}$ & 0.1184 & 0.6222 & 0.8125 & 1.0000 & 1.0000 & 1.0000 & 1.0000 & 1.0000 & 1.0000 & 1.0000 \\
\hline
\end{tabular}




\begin{tabular}{|c|c|c|c|c|c|c|c|c|c|c|c|}
\hline (5) & $\mathbf{R}$ & 0.5161 & 0.4839 & 0.5484 & 0.5161 & 0.3871 & 0.3871 & 0.3871 & 0.2903 & 0.2258 & 0.2258 \\
\cline { 2 - 11 } SD & $\mathbf{P}$ & 0.0071 & 0.0164 & 0.0217 & 0.0430 & 0.0399 & 0.0833 & 0.0764 & 0.1098 & 0.1167 & 0.2121 \\
\hline $\mathbf{( 6 )}$ & $\mathbf{R}$ & 0.2258 & 0.2258 & 0.2258 & 0.2258 & 0.2258 & 0.2258 & 0.2258 & 0.2258 & 0.2258 & 0.2258 \\
\cline { 2 - 11 } SD+STM-MH & $\mathbf{P}$ & 1.0000 & 1.0000 & 1.0000 & 1.0000 & 1.0000 & 1.0000 & 1.0000 & 1.0000 & 1.0000 & 1.0000 \\
\hline $\mathbf{( 7 )}$ & $\mathbf{R}$ & 0.5806 & 0.5806 & 0.5806 & 0.5806 & 0.5806 & 0.5806 & 0.5806 & 0.5806 & 0.5806 & 0.5806 \\
\cline { 2 - 11 } HZ & $\mathbf{P}$ & 0.5806 & 0.5806 & 0.5806 & 0.5806 & 0.5806 & 0.5806 & 0.5806 & 0.5806 & 0.5806 & 0.5806 \\
\hline
\end{tabular}

Table 6. The precision and recall rates for resolution change transformation.

\begin{tabular}{|c|c|c|c|c|c|c|c|c|c|c|c|}
\hline & & $k=10$ & $k=20$ & $k=30$ & $k=40$ & $k=50$ & $k=60$ & $k=70$ & $k=80$ & $k=90$ & $k=100$ \\
\hline \multirow{2}{*}{$\begin{array}{c}(1) \\
\text { OM+STM }\end{array}$} & $\mathbf{R}$ & 1.0000 & 0.9355 & 0.9355 & 0.7419 & 0.5806 & 0.2581 & 0.2581 & 0.2581 & 0.2581 & 0.2581 \\
\hline & $\mathbf{P}$ & 0.3229 & 0.6042 & 0.7436 & 1.0000 & 1.0000 & 1.0000 & 1.0000 & 1.0000 & 1.0000 & 1.0000 \\
\hline \multirow{2}{*}{$\begin{array}{c}(2) \\
\text { SD+STM }\end{array}$} & $\mathbf{R}$ & 0.5484 & 0.4839 & 0.5484 & 0.6129 & 0.6452 & 0.6129 & 0.5806 & 0.5806 & 0.6129 & 0.6129 \\
\hline & $\mathbf{P}$ & 0.8947 & 0.9375 & 0.9444 & 0.9048 & 0.9091 & 0.8636 & 0.9474 & 0.9000 & 0.9048 & 0.9048 \\
\hline \multirow{2}{*}{$\begin{array}{c}\text { (3) } \\
\mathrm{OM}+\mathrm{SD}+\mathrm{STM}\end{array}$} & $\mathbf{R}$ & 0.9032 & 0.9032 & 0.9032 & 0.9677 & 0.9355 & 0.9355 & 0.9355 & 0.9355 & 0.9355 & 0.9355 \\
\hline & $\mathbf{P}$ & 0.9032 & 0.9655 & 0.9655 & 0.9375 & 0.9063 & 0.9063 & 0.9355 & 0.9063 & 0.9355 & 0.9355 \\
\hline \multirow{2}{*}{$\begin{array}{l}(4) \\
\text { OM }\end{array}$} & $\mathbf{R}$ & 1.0000 & 0.9355 & 0.9355 & 0.7419 & 0.5806 & 0.2581 & 0.2581 & 0.2581 & 0.2581 & 0.2581 \\
\hline & $\mathbf{P}$ & 0.1225 & 0.5472 & 0.6905 & 0.9583 & 1.0000 & 1.0000 & 1.0000 & 1.0000 & 1.0000 & 1.0000 \\
\hline \multirow{2}{*}{$\begin{array}{l}5) \\
\text { SD }\end{array}$} & $\mathbf{R}$ & 0.6774 & 0.7097 & 0.7419 & 0.7742 & 0.7419 & 0.6452 & 0.6452 & 0.6452 & 0.6452 & 0.6774 \\
\hline & $\mathbf{P}$ & 0.0085 & 0.0199 & 0.0237 & 0.0519 & 0.0535 & 0.0794 & 0.0680 & 0.1005 & 0.0990 & 0.1214 \\
\hline \multirow{2}{*}{$\begin{array}{c}\text { (6) } \\
\text { SD+STM-MH } \\
\end{array}$} & $\mathbf{R}$ & 0.5806 & 0.5806 & 0.5806 & 0.5806 & 0.5806 & 0.5806 & 0.5806 & 0.5806 & 0.5806 & 0.5806 \\
\hline & $\mathbf{P}$ & 1.0000 & 1.0000 & 1.0000 & 1.0000 & 1.0000 & 1.0000 & 1.0000 & 1.0000 & 1.0000 & 1.0000 \\
\hline \multirow{2}{*}{$\begin{array}{l}(7) \\
\text { HZ } \\
\end{array}$} & $\mathbf{R}$ & 0.8710 & 0.8710 & 0.8710 & 0.8710 & 0.8710 & 0.8710 & 0.8710 & 0.8710 & 0.8710 & 0.8710 \\
\hline & $\mathbf{P}$ & 0.8710 & 0.8710 & 0.8710 & 0.8710 & 0.8710 & 0.8710 & 0.8710 & 0.8710 & 0.8710 & 0.8710 \\
\hline
\end{tabular}

5.5.2 Partial region-discarded spatial transformation. This category includes cropping and zooming in. The results are shown in Tables 7 and 8.

For the ordinal-based methods, the recall rates degrade slightly in both the cropping and zooming in types. Moreover, their performances in partial region-discarded spatial transformation are not as good as those in whole region-preserved spatial transformation. This is because a frame's ordinal measure, which models the property of the whole frame region, might be totally different if the frame is modified by partial region-discarded spatial transformation. The same problem arises in Hoad and Zobel's method because its signature also models the whole frame region property. Interestingly, Hoad and Zoble's method performs poorly in the cropping type, but quite well in the zooming in type.

On the other hand, the SIFT descriptor is less affected in this transformation category. Actually, Method (2) achieves good results. Although the ordinal measure and the SIFT descriptor have both advantages and limitations, their different characteristics complement each other very well. Combining the two features not only enhances the detection accuracy, but also widens the coverage to more transformation types. Method (3) provides good evidence to support the above viewpoint.

Table 7. The precision and recall rates for cropping transformation.

\begin{tabular}{|c|c|c|c|c|c|c|c|c|c|c|c|}
\hline \multicolumn{2}{|c|}{} & $\boldsymbol{k}=\mathbf{1 0}$ & $\boldsymbol{k}=\mathbf{2 0}$ & $\boldsymbol{k}=\mathbf{3 0}$ & $\boldsymbol{k}=\mathbf{4 0}$ & $\boldsymbol{k}=\mathbf{5 0}$ & $\boldsymbol{k}=\mathbf{6 0}$ & $\boldsymbol{k}=\mathbf{7 0}$ & $\boldsymbol{k}=\mathbf{8 0}$ & $\boldsymbol{k}=\mathbf{9 0}$ & $\boldsymbol{k}=\mathbf{1 0 0}$ \\
\hline $\mathbf{( 1 )}$ & $\mathbf{R}$ & 0.6774 & 0.7742 & 0.6774 & 0.2258 & 0.0968 & 0.0323 & 0.0323 & 0.0323 & 0.0323 & 0.0323 \\
\cline { 2 - 11 } OM+STM & $\mathbf{P}$ & 0.2838 & 0.5455 & 0.8400 & 1.0000 & 1.0000 & 1.0000 & 1.0000 & 1.0000 & 1.0000 & 1.0000 \\
\hline $\begin{array}{c}(\mathbf{2}) \\
\text { SD+STM }\end{array}$ & $\mathbf{R}$ & 0.8387 & 0.8387 & 0.8065 & 0.8387 & 0.8710 & 0.8710 & 0.8710 & 0.8387 & 0.8387 & 0.8387 \\
\cline { 2 - 11 } & $\mathbf{P}$ & 0.9630 & 0.8966 & 0.8929 & 0.8966 & 0.9000 & 0.9310 & 0.9310 & 0.9630 & 0.9630 & 0.9630 \\
\hline \multirow{2}{*}{ OM+SD+STM } & $\mathbf{R}$ & $\mathbf{0 . 9 3 5 5}$ & 0.9355 & 0.9355 & 0.9032 & 0.9355 & 0.9032 & 0.9032 & 0.9032 & 0.9032 & 0.9032 \\
\cline { 2 - 11 } & $\mathbf{P}$ & $\mathbf{0 . 9 6 6 7}$ & 0.9063 & 0.9063 & 0.9032 & 0.9063 & 0.9333 & 0.9333 & 0.9655 & 0.9655 & 0.9655 \\
\hline
\end{tabular}




\begin{tabular}{|c|c|c|c|c|c|c|c|c|c|c|c|}
\hline $\mathbf{( 4 )}$ & $\mathbf{R}$ & 0.7097 & 0.7742 & 0.7097 & 0.2258 & 0.0968 & 0.0323 & 0.0323 & 0.0323 & 0.0323 & 0.0323 \\
\cline { 2 - 11 } $\mathbf{O M}$ & $\mathbf{P}$ & 0.1053 & 0.4211 & 0.8148 & 1.0000 & 1.0000 & 1.0000 & 1.0000 & 1.0000 & 1.0000 & 1.0000 \\
\hline $\mathbf{( 5 )}$ & $\mathbf{R}$ & 0.9032 & 0.8710 & 0.8710 & 0.8710 & 0.9032 & 0.9032 & 0.9032 & 0.8710 & 0.8710 & 0.8710 \\
\cline { 2 - 11 } SD & $\mathbf{P}$ & 0.0088 & 0.0190 & 0.0207 & 0.0356 & 0.0393 & 0.0625 & 0.0606 & 0.0836 & 0.0925 & 0.1084 \\
\hline $\mathbf{( 6 )}$ & $\mathbf{R}$ & 0.8065 & 0.8065 & 0.8065 & 0.8065 & 0.8065 & 0.8065 & 0.8065 & 0.8065 & 0.8065 & 0.8065 \\
\cline { 2 - 11 } SD+STM-MH & $\mathbf{P}$ & 1.0000 & 1.0000 & 1.0000 & 1.0000 & 1.0000 & 1.0000 & 1.0000 & 1.0000 & 1.0000 & 1.0000 \\
\hline $\mathbf{( 7 )}$ & $\mathbf{R}$ & 0.2903 & 0.2903 & 0.2903 & 0.2903 & 0.2903 & 0.2903 & 0.2903 & 0.2903 & 0.2903 & 0.2903 \\
\cline { 2 - 10 } $\mathbf{H Z}$ & $\mathbf{P}$ & 0.2903 & 0.2903 & 0.2903 & 0.2903 & 0.2903 & 0.2903 & 0.2903 & 0.2903 & 0.2903 & 0.2903 \\
\hline
\end{tabular}

Table 8. The precision and recall rates for zooming in transformation.

\begin{tabular}{|c|c|c|c|c|c|c|c|c|c|c|c|}
\hline & & $k=10$ & $k=20$ & $k=30$ & $k=40$ & $k=50$ & $k=60$ & $k=70$ & $k=80$ & $k=90$ & $k=100$ \\
\hline \multirow{2}{*}{$\begin{array}{c}(1) \\
\text { OM+STM }\end{array}$} & $\mathbf{R}$ & 0.6129 & 0.7742 & 0.6452 & 0.3226 & 0.1290 & 0.0323 & 0.0323 & 0.0323 & 0.0323 & 0.0323 \\
\hline & $\mathbf{P}$ & 0.2375 & 0.5714 & 0.8333 & 1.0000 & 1.0000 & 1.0000 & 1.0000 & 1.0000 & 1.0000 & 1.0000 \\
\hline \multirow{2}{*}{$\begin{array}{c}(2) \\
\text { SD+STM }\end{array}$} & $\mathbf{R}$ & 0.6129 & 0.8065 & 0.8710 & 0.8065 & 0.8387 & 0.8387 & 0.8710 & 0.8387 & 0.8065 & 0.8065 \\
\hline & $\mathbf{P}$ & 0.8636 & 0.9259 & 0.9000 & 0.9259 & 0.9286 & 0.9630 & 0.9310 & 0.9630 & 0.9615 & 0.9615 \\
\hline \multirow{2}{*}{$\begin{array}{c}\text { (3) } \\
\mathrm{OM}+\mathrm{SD}+\mathrm{STM}\end{array}$} & $\mathbf{R}$ & 0.8065 & 0.9355 & 0.9355 & 0.9355 & 0.9355 & 0.9355 & 0.9355 & 0.9032 & 0.9032 & 0.8710 \\
\hline & $\mathbf{P}$ & 0.8929 & 0.9355 & 0.9063 & 0.9355 & 0.9355 & 0.9667 & 0.9355 & 0.9655 & 0.9655 & 0.9643 \\
\hline \multirow{2}{*}{$\begin{array}{l}\text { (4) } \\
\text { OM }\end{array}$} & $\mathbf{R}$ & 0.7097 & 0.8065 & 0.6774 & 0.3226 & 0.1290 & 0.0323 & 0.0323 & 0.0323 & 0.0323 & 0.0323 \\
\hline & $\mathbf{P}$ & 0.0873 & 0.4630 & 0.7500 & 1.0000 & 1.0000 & 1.0000 & 1.0000 & 1.0000 & 1.0000 & 1.0000 \\
\hline \multirow{2}{*}{$\begin{array}{l}\text { (5) } \\
\text { SD }\end{array}$} & $\mathbf{R}$ & 0.8065 & 0.8387 & 0.9355 & 0.8387 & 0.9032 & 0.8710 & 0.9032 & 0.8387 & 0.8065 & 0.8065 \\
\hline & $\mathbf{P}$ & 0.0104 & 0.0250 & 0.0287 & 0.0466 & 0.0535 & 0.0918 & 0.0915 & 0.1171 & 0.1283 & 0.1397 \\
\hline \multirow{2}{*}{$\begin{array}{c}\mathbf{( 6 )} \\
\text { SD+STM-MH }\end{array}$} & $\mathbf{R}$ & 0.6452 & 0.6452 & 0.6452 & 0.6452 & 0.6452 & 0.6452 & 0.6452 & 0.6452 & 0.6452 & 0.6452 \\
\hline & $\mathbf{P}$ & 1.0000 & 1.0000 & 1.0000 & 1.0000 & 1.0000 & 1.0000 & 1.0000 & 1.0000 & 1.0000 & 1.0000 \\
\hline \multirow{2}{*}{$\begin{array}{l}\text { (7) } \\
\text { HZ }\end{array}$} & $\mathbf{R}$ & 0.8710 & 0.8710 & 0.8710 & 0.8710 & 0.8710 & 0.8710 & 0.8710 & 0.8710 & 0.8710 & 0.8710 \\
\hline & $\mathbf{P}$ & 0.8710 & 0.8710 & 0.8710 & 0.8710 & 0.8710 & 0.8710 & 0.8710 & 0.8710 & 0.8710 & 0.8710 \\
\hline
\end{tabular}

5.5.3 Frame number-changed temporal transformation. This category includes slow motion, fast forward, and frame rate change. The results are shown in Tables 9-11.

The slow motion and fast forward types halve and double the query video speed, respectively; thus, a 30-second source sequence becomes 60-second query clip and 15second query clip, respectively. The change in the query video's speed induces that the query content does not synchronize with the target content in the window. For the frame rate change type, Methods (1)-(6) re-sample each query video to synchronize with the target sequence's frame rate, whereas Method (7) matches the two sequence frames directly without re-sampling.

In the slow motion and frame rate change types, the performances of the ordinal and SIFT-based methods are generally similar to their performances in the brightness, compression, and noise types. However, we notice that the SIFT-based methods perform differently in the fast forward type and the previous transformation types; their accuracy distributions are more scattered in the fast forward type. In addition, Method (5)'s precision rates improve noticeably as $k$ increases in the fast forward type. We consider that with fewer feature descriptors in a sequence, $k$ has a greater effect on the accuracy. This viewpoint is also held in the ordinal-based methods, and they are even more sensitive to $k$ than the SIFT-based methods.

Hoad and Zobel's method performs poorly in this transformation category. Although the approximate string matching scheme can compensate for the minor discrepancy in the 
number of frames, the dynamic programming constraints make it ineffective when the number of frames varies greatly. Another reason is due to their proposed signature, in which the color-shift and centroid-based magnitudes are conceptually amortized in neighboring frames. If the number of frames increases or decreases substantially, the method might produce a very different signature pattern from the original.

Table 9. The precision and recall rates for slow motion transformation.

\begin{tabular}{|c|c|c|c|c|c|c|c|c|c|c|c|}
\hline & & $k=10$ & $k=20$ & $k=30$ & $k=40$ & $k=50$ & $k=60$ & $k=70$ & $k=80$ & $k=90$ & $k=100$ \\
\hline \multirow{2}{*}{$\begin{array}{c}(1) \\
\text { OM+STM }\end{array}$} & $\mathbf{R}$ & 0.8710 & 0.8710 & 0.8710 & 0.8065 & 0.7742 & 0.5806 & 0.2903 & 0.2258 & 0.0968 & 0.0000 \\
\hline & $\mathbf{P}$ & 0.5870 & 0.7500 & 0.7105 & 0.9615 & 0.8889 & 1.0000 & 1.0000 & 1.0000 & 1.0000 & 0.0000 \\
\hline \multirow{2}{*}{$\begin{array}{c}(2) \\
\text { SD+STM }\end{array}$} & $\mathbf{R}$ & 0.6774 & 0.8387 & 0.8387 & 0.8065 & 0.7742 & 0.8065 & 0.8387 & 0.8065 & 0.8065 & 0.8065 \\
\hline & $\mathbf{P}$ & 0.9130 & 1.0000 & 0.9630 & 0.9259 & 0.9231 & 0.9615 & 0.9630 & 0.9259 & 0.9259 & 0.9259 \\
\hline \multirow{2}{*}{$\begin{array}{c}\text { (3) } \\
\mathrm{OM}+\mathrm{SD}+\mathrm{STM}\end{array}$} & $\mathbf{R}$ & 0.8387 & 0.9355 & 0.9355 & 0.9355 & 0.9355 & 0.9355 & 0.9355 & 0.9355 & 0.9355 & 0.9355 \\
\hline & $\mathbf{P}$ & 0.9286 & 1.0000 & 0.9667 & 0.9355 & 0.9355 & 0.9667 & 0.9667 & 0.9355 & 0.9355 & 0.9355 \\
\hline \multirow{2}{*}{$\begin{array}{l}\text { (4) } \\
\text { OM }\end{array}$} & $\mathbf{R}$ & 0.8710 & 0.8710 & 0.8710 & 0.8065 & 0.7742 & 0.5806 & 0.2903 & 0.2258 & 0.0968 & 0.0000 \\
\hline & $\mathbf{P}$ & 0.1038 & 0.3649 & 0.5400 & 0.8621 & 0.8889 & 1.0000 & 1.0000 & 1.0000 & 1.0000 & 0.0000 \\
\hline \multirow{2}{*}{$\begin{array}{l}\text { (5) } \\
\text { SD }\end{array}$} & $\mathbf{R}$ & 0.8710 & 0.9677 & 0.9355 & 0.9355 & 0.9032 & 0.9355 & 0.9677 & 0.9355 & 0.9355 & 0.9355 \\
\hline & $\mathbf{P}$ & 0.0110 & 0.0180 & 0.0169 & 0.0215 & 0.0194 & 0.0235 & 0.0226 & 0.0241 & 0.0237 & 0.0256 \\
\hline \multirow{2}{*}{$\begin{array}{c}(6) \\
\text { SD+STM-MH }\end{array}$} & $\mathbf{R}$ & 0.6774 & 0.6774 & 0.6774 & 0.6774 & 0.6774 & 0.6774 & 0.6774 & 0.6774 & 0.6774 & 0.6774 \\
\hline & $\mathbf{P}$ & 1.0000 & 1.0000 & 1.0000 & 1.0000 & 1.0000 & 1.0000 & 1.0000 & 1.0000 & 1.0000 & 1.0000 \\
\hline \multirow{2}{*}{$\begin{array}{l}\text { (7) } \\
\text { HZ }\end{array}$} & $\mathbf{R}$ & 0.0323 & 0.0323 & 0.0323 & 0.0323 & 0.0323 & 0.0323 & 0.0323 & 0.0323 & 0.0323 & 0.0323 \\
\hline & $\mathbf{P}$ & 0.0323 & 0.0323 & 0.0323 & 0.0323 & 0.0323 & 0.0323 & 0.0323 & 0.0323 & 0.0323 & 0.0323 \\
\hline
\end{tabular}

Table 10. The precision and recall rates for fast forward transformation.

\begin{tabular}{|c|c|c|c|c|c|c|c|c|c|c|c|}
\hline & & $k=10$ & $k=20$ & $k=30$ & $k=40$ & $k=50$ & $k=60$ & $k=70$ & $k=80$ & $k=90$ & $k=100$ \\
\hline \multirow{2}{*}{$\begin{array}{c}(1) \\
\text { OM+STM }\end{array}$} & $\mathbf{R}$ & 0.8710 & 0.9032 & 0.5161 & 0.5161 & 0.5161 & 0.5161 & 0.5161 & 0.5161 & 0.5161 & 0.5161 \\
\hline & $\mathbf{P}$ & 0.3506 & 0.8750 & 1.0000 & 1.0000 & 1.0000 & 1.0000 & 1.0000 & 1.0000 & 1.0000 & 1.0000 \\
\hline \multirow{2}{*}{$\begin{array}{c}(2) \\
\text { SD+STM }\end{array}$} & $\mathbf{R}$ & 0.7097 & 0.7419 & 0.9032 & 0.9032 & 0.9032 & 0.8387 & 0.8065 & 0.7742 & 0.6129 & 0.4194 \\
\hline & $\mathbf{P}$ & 0.7586 & 0.7667 & 0.8750 & 0.9333 & 0.9032 & 0.9286 & 0.8259 & 0.9231 & 0.9048 & 0.7647 \\
\hline \multirow{2}{*}{$\begin{array}{c}\text { (3) } \\
\text { OM+SD+STM }\end{array}$} & $\mathbf{R}$ & 0.9355 & 0.9677 & 1.0000 & 1.0000 & 1.0000 & 1.0000 & 1.0000 & 1.0000 & 0.9355 & 0.8710 \\
\hline & $\mathbf{P}$ & 0.8056 & 0.8108 & 0.8857 & 0.9394 & 0.9118 & 0.9394 & 0.9394 & 0.9394 & 0.9355 & 0.8710 \\
\hline \multirow{2}{*}{$\begin{array}{l}\text { (4) } \\
\text { OM }\end{array}$} & $\mathbf{R}$ & 1.0000 & 0.9355 & 0.5484 & 0.5484 & 0.5484 & 0.5484 & 0.5484 & 0.5484 & 0.5484 & 0.5484 \\
\hline & $\mathbf{P}$ & 0.1640 & 0.8286 & 0.9444 & 0.9444 & 0.9444 & 0.9444 & 0.9444 & 0.9444 & 0.9444 & 0.9444 \\
\hline \multirow{2}{*}{$\begin{array}{l}\text { (5) } \\
\text { SD }\end{array}$} & $\mathbf{R}$ & 0.9032 & 0.8710 & 0.9677 & 0.9355 & 0.9032 & 0.8387 & 0.8065 & 0.8065 & 0.6452 & 0.4516 \\
\hline & $\mathbf{P}$ & 0.0170 & 0.0651 & 0.1031 & 0.2661 & 0.3218 & 0.5098 & 0.5814 & 0.6410 & 0.6667 & 0.6364 \\
\hline \multirow{2}{*}{$\begin{array}{c}\mathbf{( 6 )} \\
\text { SD+STM-MH }\end{array}$} & $\mathbf{R}$ & 0.6129 & 0.6129 & 0.6129 & 0.6129 & 0.6129 & 0.6129 & 0.6129 & 0.6129 & 0.6129 & 0.6129 \\
\hline & $\mathbf{P}$ & 1.0000 & 1.0000 & 1.0000 & 1.0000 & 1.0000 & 1.0000 & 1.0000 & 1.0000 & 1.0000 & 1.0000 \\
\hline \multirow{2}{*}{$\begin{array}{l}\text { (7) } \\
\mathrm{HZ}\end{array}$} & $\mathbf{R}$ & 0.2258 & 0.2258 & 0.2258 & 0.2258 & 0.2258 & 0.2258 & 0.2258 & 0.2258 & 0.2258 & 0.2258 \\
\hline & $\mathbf{P}$ & 0.2258 & 0.2258 & 0.2258 & 0.2258 & 0.2258 & 0.2258 & 0.2258 & 0.2258 & 0.2258 & 0.2258 \\
\hline
\end{tabular}

Table 11. The precision and recall rates for frame rate change transformation.

\begin{tabular}{|c|c|c|c|c|c|c|c|c|c|c|c|}
\hline & & $k=10$ & $k=20$ & $k=30$ & $k=40$ & $k=50$ & $k=60$ & $k=70$ & $k=\mathbf{8 0}$ & $k=90$ & $k=100$ \\
\hline \multirow{2}{*}{$\begin{array}{c}(1) \\
\text { OM+STM }\end{array}$} & $\mathbf{R}$ & 0.9677 & 0.9355 & 0.9355 & 0.7419 & 0.6129 & 0.2903 & 0.2903 & 0.2903 & 0.2903 & 0.2903 \\
\hline & $\mathbf{P}$ & 0.3261 & 0.6591 & 0.8529 & 0.9583 & 1.0000 & 1.0000 & 1.0000 & 1.0000 & 1.0000 & 1.0000 \\
\hline \multirow{2}{*}{$\begin{array}{c}(2) \\
\text { SD+STM }\end{array}$} & $\mathbf{R}$ & 0.9677 & 0.9677 & 0.9677 & 0.9677 & 0.9677 & 0.9677 & 0.9677 & 0.9355 & 0.9032 & 0.9032 \\
\hline & $\mathbf{P}$ & 0.8108 & 0.8824 & 0.8824 & 0.9677 & 0.9375 & 0.9677 & 0.9375 & 0.9667 & 0.9655 & 0.9655 \\
\hline \multirow{2}{*}{$\begin{array}{c}\text { (3) } \\
\text { OM+SD+STM }\end{array}$} & $\mathbf{R}$ & 0.9677 & 1.0000 & 1.0000 & 1.0000 & 1.0000 & 1.0000 & 1.0000 & 1.0000 & 1.0000 & 1.0000 \\
\hline & $\mathbf{P}$ & 0.8108 & 0.8857 & 0.8857 & 0.9688 & 0.9394 & 0.9688 & 0.9394 & 0.9688 & 0.9688 & 0.9688 \\
\hline \multirow{2}{*}{$\begin{array}{l}(4) \\
\text { OM }\end{array}$} & $\mathbf{R}$ & 1.0000 & 0.9355 & 0.9355 & 0.7419 & 0.6129 & 0.2903 & 0.2903 & 0.2903 & 0.2903 & 0.2903 \\
\hline & $\mathbf{P}$ & 0.1270 & 0.5800 & 0.7838 & 0.9200 & 0.9500 & 1.0000 & 1.0000 & 1.0000 & 1.0000 & 1.0000 \\
\hline \multirow{2}{*}{$\begin{array}{l}\text { (5) } \\
\text { SD }\end{array}$} & $\mathbf{R}$ & 1.0000 & 0.9677 & 0.9677 & 0.9677 & 0.9677 & 0.9677 & 0.9677 & 0.9355 & 0.9355 & 0.9355 \\
\hline & $\mathbf{P}$ & 0.0121 & 0.0271 & 0.0299 & 0.0541 & 0.0566 & 0.0938 & 0.0917 & 0.1283 & 0.1480 & 0.1871 \\
\hline \multirow{2}{*}{$\begin{array}{c}(6) \\
\text { SD+STM-MH }\end{array}$} & $\mathbf{R}$ & 1.0000 & 1.0000 & 1.0000 & 1.0000 & 1.0000 & 1.0000 & 1.0000 & 1.0000 & 1.0000 & 1.0000 \\
\hline & $\mathbf{P}$ & 1.0000 & 1.0000 & 1.0000 & 1.0000 & 1.0000 & 1.0000 & 1.0000 & 1.0000 & 1.0000 & 1.0000 \\
\hline \multirow{2}{*}{$\begin{array}{l}\text { (7) } \\
\text { HZ }\end{array}$} & $\mathbf{R}$ & 0.2903 & 0.2903 & 0.2903 & 0.2903 & 0.2903 & 0.2903 & 0.2903 & 0.2903 & 0.2903 & 0.2903 \\
\hline & $\mathbf{P}$ & 0.2903 & 0.2903 & 0.2903 & 0.2903 & 0.2903 & 0.2903 & 0.2903 & 0.2903 & 0.2903 & 0.2903 \\
\hline
\end{tabular}


5.5.4 Frame order-changed temporal transformation. This category includes frame swap and frame insertion/deletion. The results are shown in Tables 12 and 13.

The results of the frame swap type show that the ordinal-based and SIFT-based methods are basically unaffected by changing the frame order in a video sequence because of using the histogram-based feature representation. In the frame insertion/deletion type, the recall rates of the ordinal-based methods degrade significantly compared with those in the frame swap type; even no result is retrieved when $k \geq 60$. The influence of the SIFTbased methods is relatively limited. We consider that a larger number of feature descriptors in the histogram could provide stronger resistance when partial content is removed. This explains why the SIFT-based methods yield more robust recall rates than the ordinal-based methods in this transformation type and partial region-discarded spatial transformation.

Hoad and Zobel's method remains unsatisfactory in this transformation category because of the frame pair mapping criterion posed by approximate string matching.

Table 12. The precision and recall rates for frame swap transformation.

\begin{tabular}{|c|c|c|c|c|c|c|c|c|c|c|c|}
\hline & & $k=10$ & $k=20$ & $k=30$ & $k=40$ & $k=50$ & $k=60$ & $k=70$ & $k=80$ & $k=90$ & $k=100$ \\
\hline \multirow{2}{*}{$\begin{array}{c}(1) \\
\text { OM+STM }\end{array}$} & $\mathbf{R}$ & 1.0000 & 0.9355 & 0.9355 & 0.7419 & 0.6129 & 0.2581 & 0.2581 & 0.2581 & 0.2581 & 0.2581 \\
\hline & $\mathbf{P}$ & 0.3131 & 0.6444 & 0.8529 & 1.0000 & 1.0000 & 1.0000 & 1.0000 & 1.0000 & 1.0000 & 1.0000 \\
\hline \multirow{2}{*}{$\begin{array}{c}(2) \\
\text { SD+STM }\end{array}$} & $\mathbf{R}$ & 0.8387 & 0.9677 & 0.9677 & 0.9677 & 0.9355 & 0.9355 & 0.9355 & 0.9032 & 0.9032 & 0.9032 \\
\hline & $\mathbf{P}$ & 0.9286 & 0.9375 & 0.8824 & 0.9091 & 0.9355 & 0.9667 & 0.9667 & 0.9655 & 0.9655 & 0.9655 \\
\hline \multirow{2}{*}{$\begin{array}{c}\text { (3) } \\
\mathrm{OM}+\mathrm{SD}+\mathrm{STM} \\
\end{array}$} & $\mathbf{R}$ & 0.9355 & 0.9677 & 0.9677 & 0.9677 & 0.9677 & 0.9677 & 0.9677 & 0.9677 & 0.9677 & 0.9677 \\
\hline & $\mathbf{P}$ & 0.9355 & 0.9375 & 0.8824 & 0.9091 & 0.9375 & 0.9677 & 0.9677 & 0.9677 & 0.9677 & 0.9677 \\
\hline \multirow{2}{*}{$\begin{array}{l}\text { (4) } \\
\text { OM }\end{array}$} & $\mathbf{R}$ & 1.0000 & 0.9355 & 0.9355 & 0.7419 & 0.6129 & 0.2581 & 0.2581 & 0.2581 & 0.2581 & 0.2581 \\
\hline & $\mathbf{P}$ & 0.1308 & 0.5686 & 0.7838 & 0.9583 & 1.0000 & 1.0000 & 1.0000 & 1.0000 & 1.0000 & 1.0000 \\
\hline \multirow{2}{*}{$\begin{array}{l}\text { (5) } \\
\text { SD }\end{array}$} & $\mathbf{R}$ & 0.9677 & 1.0000 & 1.0000 & 1.0000 & 0.9677 & 0.9677 & 0.9677 & 0.9355 & 0.9355 & 0.9355 \\
\hline & $\mathbf{P}$ & 0.0110 & 0.0275 & 0.0300 & 0.0513 & 0.0566 & 0.0885 & 0.0845 & 0.1198 & 0.1343 & 0.1526 \\
\hline \multirow{2}{*}{$\begin{array}{c}\mathbf{( 6 )} \\
\text { SD+STM-MH }\end{array}$} & $\mathbf{R}$ & 0.9677 & 0.9677 & 0.9677 & 0.9677 & 0.9677 & 0.9677 & 0.9677 & 0.9677 & 0.9677 & 0.9677 \\
\hline & $\mathbf{P}$ & 1.0000 & 1.0000 & 1.0000 & 1.0000 & 1.0000 & 1.0000 & 1.0000 & 1.0000 & 1.0000 & 1.0000 \\
\hline \multirow{2}{*}{$\begin{array}{l}\text { (7) } \\
\text { HZ }\end{array}$} & $\mathbf{R}$ & 0.4516 & 0.4516 & 0.4516 & 0.4516 & 0.4516 & 0.4516 & 0.4516 & 0.4516 & 0.4516 & 0.4516 \\
\hline & $\mathbf{P}$ & 0.4516 & 0.4516 & 0.4516 & 0.4516 & 0.4516 & 0.4516 & 0.4516 & 0.4516 & 0.4516 & 0.4516 \\
\hline
\end{tabular}

Table 13. The precision and recall rates for frame insertion/deletion transformation.

\begin{tabular}{|c|c|c|c|c|c|c|c|c|c|c|c|}
\hline & & $k=10$ & $k=20$ & $k=30$ & $k=40$ & $k=50$ & $k=60$ & $k=70$ & $k=80$ & $k=90$ & $k=100$ \\
\hline \multirow{2}{*}{$\begin{array}{c}(1) \\
\text { OM+STM }\end{array}$} & $\mathbf{R}$ & 0.7419 & 0.7742 & 0.6452 & 0.2258 & 0.0968 & 0.0000 & 0.0000 & 0.0000 & 0.0000 & 0.0000 \\
\hline & $\mathbf{P}$ & 0.6053 & 1.0000 & 1.0000 & 1.0000 & 1.0000 & 0.0000 & 0.0000 & 0.0000 & 0.0000 & 0.0000 \\
\hline \multirow{2}{*}{$\begin{array}{c}(2) \\
\text { SD+STM } \\
\end{array}$} & $\mathbf{R}$ & 0.6452 & 0.6452 & 0.6774 & 0.6774 & 0.7097 & 0.5806 & 0.6129 & 0.8506 & 0.6129 & 0.6452 \\
\hline & $\mathbf{P}$ & 0.8696 & 0.9524 & 0.9545 & 0.9545 & 0.9565 & 0.9474 & 0.9500 & 0.9474 & 0.9500 & 0.9524 \\
\hline \multirow{2}{*}{$\begin{array}{c}\text { (3) } \\
\mathrm{OM}+\mathrm{SD}+\mathrm{STM}\end{array}$} & $\mathbf{R}$ & 0.9677 & 0.9355 & 0.9677 & 0.9677 & 0.9355 & 0.9032 & 0.9355 & 0.9032 & 0.9032 & 0.9032 \\
\hline & $\mathbf{P}$ & 0.9091 & 0.9667 & 0.9667 & 0.9667 & 0.9667 & 0.9655 & 0.9667 & 0.9655 & 0.9655 & 0.9655 \\
\hline \multirow{2}{*}{$\begin{array}{l}\text { (4) } \\
\text { OM }\end{array}$} & $\mathbf{R}$ & 0.8710 & 0.8387 & 0.6452 & 0.2258 & 0.0968 & 0.0000 & 0.0000 & 0.0000 & 0.0000 & 0.0000 \\
\hline & $\mathbf{P}$ & 0.2250 & 0.9286 & 0.9524 & 1.0000 & 1.0000 & 0.0000 & 0.0000 & 0.0000 & 0.0000 & 0.0000 \\
\hline \multirow{2}{*}{$\begin{array}{l}\text { (5) } \\
\text { SD }\end{array}$} & $\mathbf{R}$ & 0.9355 & 0.8387 & 0.9355 & 0.9355 & 0.9032 & 0.8387 & 0.8710 & 0.7742 & 0.7097 & 0.7097 \\
\hline & $\mathbf{P}$ & 0.0172 & 0.0563 & 0.1032 & 0.3222 & 0.3544 & 0.5778 & 0.6136 & 0.7059 & 0.8462 & 0.9167 \\
\hline \multirow{2}{*}{$\begin{array}{c}\mathbf{( 6 )} \\
\text { SD+STM-MH }\end{array}$} & $\mathbf{R}$ & 0.6774 & 0.6774 & 0.6774 & 0.6774 & 0.6774 & 0.6774 & 0.6774 & 0.6774 & 0.6774 & 0.6774 \\
\hline & $\mathbf{P}$ & 1.0000 & 1.0000 & 1.0000 & 1.0000 & 1.0000 & 1.0000 & 1.0000 & 1.0000 & 1.0000 & 1.0000 \\
\hline \multirow{2}{*}{$\begin{array}{r}\mathbf{( 7 )} \\
\mathrm{HZ} \\
\end{array}$} & $\mathbf{R}$ & 0.2581 & 0.2581 & 0.2581 & 0.2581 & 0.2581 & 0.2581 & 0.2581 & 0.2581 & 0.2581 & 0.2581 \\
\hline & $\mathbf{P}$ & 0.2581 & 0.2581 & 0.2581 & 0.2581 & 0.2581 & 0.2581 & 0.2581 & 0.2581 & 0.2581 & 0.2581 \\
\hline
\end{tabular}


5.5.5 Summary. The above experiment results indicate that, for every type of spatial and temporal transformation, Method (3) consistently outperforms all methods compared in the experiments. It yields excellent accuracy with very high recall and precision rates. The insensitivity to $k$ is another good characteristic of the combined method, which manifests that a stable and effective performance can be achieved without paying much attention to tuning up the signature length of a video sequence. To summarize, we demonstrate a promising result by integrating the min-hashing signature of complementary features, i.e., the ordinal measure and SIFT descriptor, into the spatio-temporal matching scheme.

\subsection{Computation Time}

The following computation cost evaluation was run in an environment where all the feature data of the query and target videos was extracted and loaded into the memory. We take the brightness transformation type as an example for illustration.

First, we assess the effectiveness of using histogram pruning. We define the histogram pruning ratio metric as:

$$
\text { histogram pruning ratio }=\frac{\text { the number of frames scanned in the target sequence }}{\text { the number of frames in the target sequence }} .
$$

In this metric, a lower ratio means more frames are skipped without examination in the scanning process. The metric is independent on whether spatio-temporal matching is applied or not. The histogram pruning ratios versus different $k$ for Methods (1)-(6) are listed in Table 14. The ordinal-based methods have the lowest ratio, while the combination method has the highest ratio. Generally speaking, the ratios decrease gradually as $k$ grows. This is because 1) the maximum increment for the sliding window, i.e., $g / k$, decreases as $k$ increases; and 2) using a higher $k$ in the min-hashing similarity measurement usually results in a lower similarity score averagely. It is clear from Equation (8) that the above two reasons drive the sliding window to skip more frames. Method (6) has a relatively lower ratio than Method (2) since the Jaccard similarity of two sequences is usually lower than the associated min-hashing similarity. Hence, more frames would be skipped according to Equation (8).

We define another metric, called the candidate ratio, as:

candidate ratio $=\frac{\text { the number of frames of all candidates }}{\text { the number of frames in the target sequence }}$.

This metric calculates how many candidates are selected from the target sequence. A lower ratio means that fewer candidates are selected. The candidate ratios versus $k$ for Methods (1)-(6) are listed in Table 15. Since Methods (4) and (5) do not apply spatio- 
temporal matching, they do not verify any candidate; thus their candidate ratios are zero. For Methods (1)-(3), the increase in $k$ usually reduces the number of candidates that fulfill the threshold criterion. The candidate ratio of Method (6) is much lower than that of Method (2). In other words, applying min-hashing indexing yields more candidates for later spatio-temporal matching.

Recall the inverted indexing technique in Section 4.3.1. We evaluate the time cost required to insert frames and accumulate the number of frame pairs in the inverted table. In our case, the insertion frequency of a candidate sequence is 344.01 averagely, and the number of frame pairs found in the inverted table is 7766.02 averagely. The use of inverted indexing can thus save $96.46 \%$ of the computation overhead compared with the all-pair frame similarity computation.

The time costs versus $k$ for Methods (1)-(6) are listed in Table 16. The increment of $k$ increases the computation cost for similarity measurement, but decreases the histogram pruning and candidate ratios simultaneously. For Method (3), the fastest speed occurs when $k=40$ and 50; it requires 62 milliseconds to search in a 6.1 hour video sequence. It is interesting that a higher $k$ does not ensure better degree of accuracy and might rebound on the computation cost. A suitable choice of $k$ for this video dataset would be from 30 to 90, which yields the best balance of robustness and efficiency. Consider Methods (2) and (6) that are with/without min-hashing indexing, respectively. The computation time of Method (2) is much shorter than that of Method (6). Therefore, the compact min-hashing signature significantly reduces the computation cost without significantly degrading the accuracy. In the experiments, spatio-temporal matching took averagely 0.5 and 0.8 milliseconds to verify a candidate in Methods (1) and (2), respectively. Obviously, spatiotemporal matching is worth the little extra time because it can improve the precision rate considerably.

Table 14. The histogram pruning ratio versus $k$ for Methods (1)-(6).

\begin{tabular}{|l|c|c|c|c|c|c|c|c|c|c|}
\hline & $\boldsymbol{k}=\mathbf{1 0}$ & $\boldsymbol{k}=\mathbf{2 0}$ & $\boldsymbol{k}=\mathbf{3 0}$ & $\boldsymbol{k}=\mathbf{4 0}$ & $\boldsymbol{k}=\mathbf{5 0}$ & $\boldsymbol{k}=\mathbf{6 0}$ & $\boldsymbol{k}=\mathbf{7 0}$ & $\boldsymbol{k}=\mathbf{8 0}$ & $\boldsymbol{k}=\mathbf{9 0}$ & $\boldsymbol{k}=\mathbf{1 0 0}$ \\
\hline (1) OM+STM & 0.2946 & 0.1588 & 0.1064 & 0.0779 & 0.0610 & 0.0489 & 0.0489 & 0.0489 & 0.0489 & 0.0489 \\
\hline (2) SD+STM & 0.4435 & 0.3686 & 0.2577 & 0.2060 & 0.1641 & 0.1777 & 0.1837 & 0.1642 & 0.1682 & 0.1559 \\
\hline (3) OM+SD+STM & 0.7381 & 0.5274 & 0.3641 & 0.2839 & 0.2251 & 0.2266 & 0.2326 & 0.2131 & 0.2171 & 0.2048 \\
\hline (4) OM & 0.2946 & 0.1588 & 0.1064 & 0.0779 & 0.0610 & 0.0489 & 0.0489 & 0.0489 & 0.0489 & 0.0489 \\
\hline (5) SD & 0.4435 & 0.3686 & 0.2577 & 0.2060 & 0.1641 & 0.1777 & 0.1837 & 0.1642 & 0.1682 & 0.1559 \\
\hline (6) SD+STM-MH & 0.0911 & 0.0911 & 0.0911 & 0.0911 & 0.0911 & 0.0911 & 0.0911 & 0.0911 & 0.0911 & 0.0911 \\
\hline
\end{tabular}

Table 15. The candidate ratio versus $k$ for Methods (1)-(6).

\begin{tabular}{|l|c|c|c|c|c|c|c|c|c|c|}
\hline & $\boldsymbol{k}=\mathbf{1 0}$ & $\boldsymbol{k}=\mathbf{2 0}$ & $\boldsymbol{k}=\mathbf{3 0}$ & $\boldsymbol{k}=\mathbf{4 0}$ & $\boldsymbol{k}=\mathbf{5 0}$ & $\boldsymbol{k}=\mathbf{6 0}$ & $\boldsymbol{k}=\mathbf{7 0}$ & $\boldsymbol{k}=\mathbf{8 0}$ & $\boldsymbol{k}=\mathbf{9 0}$ & $\boldsymbol{k}=\mathbf{1 0 0}$ \\
\hline (1) OM+STM & 0.0101 & 0.0022 & 0.0016 & 0.0011 & 0.0008 & 0.0003 & 0.0003 & 0.0003 & 0.0003 & 0.0003 \\
\hline (2) SD+STM & 0.1245 & 0.0512 & 0.0447 & 0.0250 & 0.0231 & 0.0133 & 0.0137 & 0.0097 & 0.0092 & 0.0078 \\
\hline (3) OM+SD+STM & 0.1346 & 0.0535 & 0.0463 & 0.0261 & 0.0239 & 0.0136 & 0.0140 & 0.0100 & 0.0095 & 0.0081 \\
\hline (4) OM & 0.0000 & 0.0000 & 0.0000 & 0.0000 & 0.0000 & 0.0000 & 0.0000 & 0.0000 & 0.0000 & 0.0000 \\
\hline
\end{tabular}




\begin{tabular}{|l|l|l|l|l|l|l|l|l|l|l|}
\hline (5) SD & 0.0000 & 0.0000 & 0.0000 & 0.0000 & 0.0000 & 0.0000 & 0.0000 & 0.0000 & 0.0000 & 0.0000 \\
\hline (6) SD+STM-MH & 0.0017 & 0.0017 & 0.0017 & 0.0017 & 0.0017 & 0.0017 & 0.0017 & 0.0017 & 0.0017 & 0.0017 \\
\hline
\end{tabular}

Table 16. The time cost (in second) versus $k$ for Methods (1)-(6).

\begin{tabular}{|l|l|l|l|l|l|l|l|l|l|l|}
\hline & $\boldsymbol{k}=\mathbf{1 0}$ & $\boldsymbol{k}=\mathbf{2 0}$ & $\boldsymbol{k}=\mathbf{3 0}$ & $\boldsymbol{k}=\mathbf{4 0}$ & $\boldsymbol{k}=\mathbf{5 0}$ & $\boldsymbol{k}=\mathbf{6 0}$ & $\boldsymbol{k}=\mathbf{7 0}$ & $\boldsymbol{k}=\mathbf{8 0}$ & $\boldsymbol{k}=\mathbf{9 0}$ & $\boldsymbol{k}=\mathbf{1 0 0}$ \\
\hline (1) OM+STM & 0.035 & 0.017 & 0.014 & 0.011 & 0.010 & 0.009 & 0.009 & 0.009 & 0.009 & 0.009 \\
\hline (2) SD+STM & 0.121 & 0.089 & 0.072 & 0.053 & 0.042 & 0.050 & 0.068 & 0.057 & 0.076 & 0.064 \\
\hline (3) OM+SD+STM & 0.145 & 0.089 & 0.087 & 0.062 & 0.062 & 0.070 & 0.095 & 0.096 & 0.109 & 0.109 \\
\hline (4) OM & 0.031 & 0.016 & 0.013 & 0.010 & 0.010 & 0.009 & 0.009 & 0.008 & 0.008 & 0.008 \\
\hline (5) SD & 0.050 & 0.062 & 0.047 & 0.035 & 0.029 & 0.042 & 0.057 & 0.048 & 0.068 & 0.057 \\
\hline (6) SD+STM-MH & 1.423 & 1.423 & 1.423 & 1.423 & 1.423 & 1.423 & 1.423 & 1.423 & 1.423 & 1.423 \\
\hline
\end{tabular}

\section{CONCLUSION}

To achieve fast and robust video copy detection, we propose a novel method that is appropriate for dealing with a variety of video transformation in a continuous video sequence. The method utilizes the min-hashing signature to represent a video sequence, and spatio-temporal matching to evaluate the content similarity between two video sequences. In addition, we employ histogram pruning and inverted indexing techniques to speed up the search process. The results of extensive experiments demonstrate the abilities of the ordinal measure and the SIFT descriptor, the impact of the min-hashing signature, the effectiveness of the spatio-temporal matching scheme, and the efficiency of the speed-up techniques. The results are very promising for a number of reasons. Specifically, the two feature descriptors complement each other quite well; the compact min-hashing signature efficiently reduces the computation cost; the spatio-temporal matching scheme effectively improves the accuracy; and the speed-up techniques accelerate the search process with great expedition. The successful integration of these factors ensures that the proposed video copy detection method is both fast and robust.

\section{ACKNOWLEDGMENTS}

The authors would like to thank the anonymous reviewers for their thoughtful comments and suggestions that have advanced the quality of this article.

\section{REFERENCES}

ANDONI, A. AND INDYK, P. 2008. Near-optimal hashing algorithms for approximate nearest neighbor in high dimension. Communications of the ACM 51, 1, 117-122.

BUHLER, J. 2001. Efficient large-scale sequence comparison by locality-sensitive hashing. Bioinformatics 17, 5, 419-218.

CHANG, S. F., CHEN, W., MENG, H. J., SUNDARAM, H., AND ZHONG, D. 1998. A fully automated content-based video search engine supporting spatiotemporal queries. IEEE Transactions on Circuits and Systems for Video Technology 8, 5, 602-615.

CHEUNG, S. C. AND ZAKHOR, A. 2003. Efficient video similarity measurement with video signature. IEEE Transactions on Circuits and Systems for Video Technology 13, 1, 59-74.

CHIU, C. Y., YANG, C. C., AND CHEN, C. S. 2007. Efficient and effective video copy detection based on spatiotemporal analysis. In Proceedings of the IEEE International Symposium on Multimedia (ISM), Taichung, Taiwan, Dec. 10-12, 202-209. 
CHIU, C. Y., CHEN, C. S., AND CHIEN, L. F. 2008. A framework for handling spatiotemporal variations in video copy detection. IEEE Transactions on Circuits and Systems for Video Technology 18, 3, 412-417.

COHEN, E., DATAR, M., FUJIWARA, S., GIONIS, A., INDYK, P., MOTWANI, R., ULLMAN, J., AND YANG, C. 2000. Finding interesting associations without support pruning. In Proceedings of the IEEE International Conference on Data Engineering (ICDE), San Diego, USA, Feb. 28-Mar. 3, 489-500.

DAS, A., DATAR, M., AND GARG, A. 2007. Google news personalization: scalable online collaborative filtering. In Proceedings of International World Wide Web Conference (WWW), Banff, Canada, May 8-12.

DEMENTHON, D. AND DOERMANN, D. 2006. Video retrieval of near-duplicates using k-nearest neighbor retrieval of spatio-temporal descriptors. Multimedia Tools and Applications 30, 3, 229-253.

DENG, Y. AND MANJUNATH, B. S. 1998. NeTra-V: toward an object-based video representation. IEEE Transactions on Circuits and Systems for Video Technology 8, 5, 616-627.

ENNESSER, F. AND MEDIONI, G. 1995. Finding Waldo, or focus of attention using local color information. IEEE Transactions on Pattern Analysis and Machine Intelligence 17, 8, 805-809.

HAMPAPUR, A. AND BOLLE, R. M. 2001. Comparison of distance measures for video copy detection. In Proceedings of the IEEE International Conference on Multimedia and Expo (ICME), Tokyo, Japan, Aug. 22-25, 737-740.

HOAD, T. C. AND ZOBEL, J. 2006. Detection of video sequence using compact signatures. ACM Transactions on Information System 24, 1, 1-50.

HUA, X. S., CHEN, X., AND ZHANG, H. J. 2004. Robust video signature based on ordinal measure. In Proceedings of the IEEE International Conference on Image Processing (ICIP), Singapore, Oct. 24-27, Volume 1, 685-688.

JAIN, A. K., VAILAYA, A., AND XIONG, W. 1999. Query by video clip. Multimedia Systems 7, 5, 369-384.

JOLY, A., BUISSON, O., AND FRELICOT, C. 2007. Content-based copy retrieval using distortion-based probabilistic similarity search. IEEE Transactions on Multimedia 9, 2, 293-306.

KASHINO, K., KUROZUMI, T., AND MURASE, H. 2003. A quick search method for audio and video signals based on histogram pruning. IEEE Transactions on Multimedia 5, 3, 348-357.

KE, Y., SUKTHANKAR, R., AND HUSTON, L. 2004. An efficient parts-based near-duplicate and sub-image retrieval system. In Proceedings of the ACM International Conference on Multimedia (MM), New York, USA, Oct. 10-16, 869-876.

KIM, C. AND VASUDEV, B. 2005. Spatiotemporal sequence matching for efficient video copy detection. IEEE Transactions on Circuits and Systems for Video Technology 15, 1, 127-132.

KIM, H. S., LEE, J., LIU, H., AND LEE, D. 2008. Video linkage: group based copied video detection. In Proceedings of the ACM International Conference on Content-based Image and Video Retrieval (CIVR), Niagara Falls, Canada, Jul. 7-9, 397-406.

LAW-TO, J., BUISSON, O., GOUET-BRUNET, V., AND BOUJEMAA, N. 2006. Robust voting algorithm based on labels of behavior for video copy detection. In Proceedings of the ACM International Conference on Multimedia (MM), Santa Barbara, CA, USA, Oct. 23-27, 835-844.

LAW-TO, J., CHEN, L., JOLY, A., LAPTEV, I., BUISSION, O., GOUET-BRUNET, V., BOUJEMAA, N., AND STENTIFORD, F. 2007. Video copy detection: a comparative study. In Proceedings of the ACM International Conference on Image and Video Retrieval (CIVR), Amsterdam, The Netherlands, Jul. 9-11, 371-378.

LIU, T., ZHANG, H. J., AND QI, F. 2003. A novel video key-frame extraction algorithm based on perceived motion energy model. IEEE Transactions on Circuits and Systems for Video Technology 13, 10, 1006-1013.

LOWE, D. G. Distinctive image features from scale-invariant keypoints. 2004. International Journal of Computer Vision 60, 2, 91-110.

MASSOUDI, A., LEFEBVRE, F., DEMARTY, C. H., OISEL, L., AND CHUPEAU, B. 2006. A video fingerprint based on visual digest and local fingerprints. In Proceedings of the IEEE International Conference on Image Processing (ICIP), Atlanta, USA, Oct. 8-11, 2297-2300.

NAPHADE, M. R. AND HUANG, T. S. 2001. A probabilistic framework for semantic video indexing, filtering, and retrieval. IEEE Transactions on Multimedia 3, 1, 141-151.

POULLOT, S., CRUCIANU, M., AND BUISSON, O. 2008. Scalable mining of large video databases using copy detection. In Proceedings of the ACM International Conference on Multimedia (MM), Vancouver, Canada, Oct. 26-31, 61-70.

SAYOOD, K. 1996. Introduction to Data Compression, Morgan Kaufmann, Los Altos, CA, USA.

SCHMID, C. AND MOHR, R. 1997. Local grayvalue invariants for image retrieval. IEEE Transactions on Pattern Analysis and Machine Intelligence 19, 5, 530-535.

SHEN, H. T., OOI, B. C., ZHOU, X., AND HUANG, Z. 2005. Towards effective indexing for very large video sequence database. In Proceedings of the ACM International Conference on Management of Data (SIGMOD), Baltimore, Maryland, USA, Jun. 14-16, 730-741.

SMOLIAR, S. W. AND ZHANG, H. 1994. Content-based video indexing and retrieval. IEEE Multimedia 1, 2 , 62-72.

SONKA, M., HLAVAC, V., AND BOYLE, R. 1999. Image Processing, Analysis, and Machine Vision, Brooks/Cole Publishing, Pacific Grove, CA, USA.

SWAIN, M. J. AND BALLARD, D. H. 1991. Color indexing. International Journal of Computer Vision 7. 1, 11-32. 
WILLEMS, G., TUYTELAARS, T., AND GOOL, L. V. 2008. Spatio-temporal features for robust contentbased video copy detection. In Proceedings of the ACM International Conference on Multimedia Information Retrieval, Vancouver, Canada, Oct. 30-31, 283-290.

WU, X., HAUPTMANN, A. G., AND NGO, C. W. 2007. Practical elimination of near-duplicates from Web video search. In Proceedings of the ACM International Conference on Multimedia (MM), Augsburg, Bavaria, Germany, Sep. 23-28. 218-227.

YUAN, J., DUAN, L. Y., TIAN, Q., AND XU, C. 2004. Fast and robust search short video clip search using an index structure. In Proceedings of the ACM International Workshop on Multimedia Information Retrieval (MIR), New York, USA, Oct. 15-16, 61-68. 